\title{
Matrix Methods for the Calculation of Stability Diagrams in Quadrupole Mass Spectrometry
}

\author{
N. V. Konenkov and M. Sudakov* \\ Department of General Physics, Ryazan State Pedagogical University, Ryazan, Russia \\ D. J. Douglas \\ Department of Chemistry, University of British Columbia, Vancouver, British Columbia, Canada
}

\begin{abstract}
The theory of the computer calculation of the stability of ion motion in periodic quadrupole fields is considered. A matrix approach for the numerical solution of the Hill equation and examples of calculations of stability diagrams are described. The advantage of this method is that it can be used for any periodic waveform. The stability diagrams with periodic rectangular waveform voltages are calculated with this approach. Calculations of the conventional stability diagram of the 3-D ion trap and the first six regions of stability of a mass filter with this method are presented. The stability of the ion motion for the case of a trapping voltage with two or more frequencies is also discussed. It is shown that quadrupole excitation with the rational angular frequency $\omega=N \Omega / P$ (where $N, P$ are integers and $\Omega$ is the angular frequency of the trapping field) leads to splitting of the stability diagram along iso- $\beta$ lines. Each stable region of the unperturbed diagram splits into $P$ stable bands. The widths of the unstable resonance lines depend on the amplitude of the auxiliary voltage and the frequency. With a low auxiliary frequency splitting of the stability diagram is greater near the boundaries of the unperturbed diagram. It is also shown that amplitude modulation of the trapping RF voltage by an auxiliary signal is equivalent to quadrupole excitation with three frequencies. The effect of modulation by a rational frequency is similar to the case of quadrupole excitation, although splitting of the stability diagram differs to some extent. The methods and results of these calculations will be useful for studies of higher stability regions, resonant excitation, and non-sinusoidal trapping voltages. (J Am Soc Mass Spectrom 2002, 13, 597-613) (C) 2002 American Society for Mass Spectrometry
\end{abstract}

$\mathrm{T}$ The diagram for the stability of ion motion is central to any discussion of the operation of quadrupole ion traps or mass filters. In quadrupole radio frequency (RF) fields, "unstable" ion motion means that the amplitude of ion oscillation increases exponentially with time and an ion is lost from the trap or filter. "Stable" motion means that the amplitude remains finite and an ion is confined. Generally both $\mathrm{DC}$ and RF voltages are used, and for a pure quadrupole potential this leads to the Mathieu equation for ion motion. The stability diagram for this equation was published more than 75 years ago [1]. The calculation methods are described in detail in the book of

Published online April 29, 2002

Address reprint requests to Dr. N. V. Konenkov, Department of General Physics, Ryazan State Pedagogical University, Svoboda Str. 49, 390000 Ryazan, Russia. E-mail: konenkov@ttc.ryazan.ru

*Current address: Shimadzu Research Laboratories (Europe) Ltd., Wharfside, Trafford Wharf Road, Manchester, M17 1GP, UK.
McLachlan [2]. Application of the theory of the Mathieu equation to quadrupole ion traps and mass filters has been discussed in monographs [3, 4]. Despite this long history, calculation of the stability of ion motion in RF quadrupole fields remains of interest for several new areas of mass spectrometry. These include the use of higher stability regions [5], application of resonant excitation [6], and the use of non-sinusoidal trapping voltages [7].

This paper discusses a general approach to the calculation of stability diagrams. The stability of ion motion is investigated with the use of the general theory of ion motion in periodic fields. The most general differential equation describing ion motion is known as the Hill equation. The stability conditions can be derived from the calculation of two independent solutions of this equation through a single cycle of the $\mathrm{RF}$ voltage. All calculations are performed by matrix methods. An advantage of this approach is that it can be 
applied to any periodic waveform, and is not limited to cases described by the Mathieu equation.

The paper is organized as follows. The equations of ion motion in the quadrupole fields of traps and mass filters are discussed in the next section. The properties of the ion motion, which follow from the general theory of linear differential equations with periodic coefficients, are then described briefly. This is followed by a description of matrix methods for the solution of the Hill and Mathieu equations. New examples of stability diagram calculations for the case of rectangular waveform voltages, the conventional Mathieu stability diagram for motion in one dimension, and stability diagrams of both the ion trap and mass filter, are presented. Calculations of ion stability with auxiliary quadrupole excitation and modulation are also shown. This is followed by a general discussion of the new results derived here. An appendix gives a brief review of the matrix mathematics used in this article.

\section{Equations of Ion Motion}

In mass spectrometry, time varying quadrupole fields are used to confine ions. The time dependent electric potential may be expressed as follows:

$$
\phi(x, y, z, t)=V(t) \cdot \Phi_{2}(x, y, z)
$$

Here $V(t)$ is a periodic trapping voltage and $\Phi_{2}(x, y, z)$ is the spatial dependence of the quadrupole potential. In a suitable Cartesian coordinate system $\Phi_{2}(x, y, z)$ for the linear mass filter and 3-D ion trap is expressed as:

$$
\begin{aligned}
& \Phi_{2}^{\text {filter }}(x, y, z)=\frac{x^{2}-y^{2}}{r_{0}^{2}} \text { or } \\
& \Phi_{2}^{\operatorname{trap}}(x, y, z)=\frac{2 z^{2}-x^{2}-y^{2}}{r_{0}^{2}} .
\end{aligned}
$$

Here $r_{0}$ is the field radius (the distance from the center of a trap to the ring electrode or the distance from the center of a mass filter to a rod). In this article the influence of field imperfections, Coulomb interactions, and ion collisions with buffer gas are not considered. All of these lead to shifts in ion oscillation frequencies, to coupling of the $x, y$, and $z$ motion, and to nonlinear phenomena. We are interested in the new properties of the ion motion, which appear with the use of nonsinusoidal periodic voltages, the use of trapping voltages that contain two or more frequencies, and the use of higher regions of stability of a mass filter. For this reason we consider the ion motion in a pure quadrupole field. The force on an ion in direction $u$, is $e Z_{i} E_{u}$, where $e Z_{i}$ is the charge on an ion and $E_{u}$ is the component of the electric field in direction $u$ given by $\mathrm{E}_{\mathrm{u}}=-\partial \phi(x, y, z, t) / \partial u$. From eq 2 the ion motions in different directions are independent and the electric force is linearly proportional to the ion position. The equation of motion (Newton's law) is

$$
M \frac{d^{2} u}{d t^{2}}=-e Z_{i} V(t) \frac{\partial \Phi}{\partial u}, \quad u=(x, y, z),
$$

where $M$ is the ion mass. A dimensionless time scale, $\xi=\Omega t / 2$, based on $\Omega$, the main trapping RF angular frequency, is usually used for the solution of eq 3 which can be written

$$
\begin{aligned}
& \frac{d^{2} u}{d \xi^{2}}+f_{u}(\xi) \cdot u=0 \\
& f_{u}(\xi)=\sigma_{u} \frac{4 e Z_{i}}{M r_{0}^{2} \Omega^{2}} V(2 \xi / \Omega), \quad f_{u}(\xi+T)=f_{u}(\xi) .
\end{aligned}
$$

$\left(\sigma_{x}, \sigma_{y}, \sigma_{z}\right)=(-2,-2,4)$ in the case of an ion trap, and $\left(\sigma_{x}, \sigma_{y}, \sigma_{z}\right)=(2,-2,0)$ for a linear mass filter. The function $f_{u}(\xi)$ is periodic with period $T$.

In the simplest case $T$ is equal to the period of the main trapping RF, which occurs when $\xi=\pi$ in dimensionless units. However the theory can be applied to trapping voltages that contain two frequencies where both frequencies are related by a rational fraction. For example, in the case of quadrupole excitation, where the auxiliary AC voltage has frequency $\omega=(N / P) \Omega$ with $N$ and $P$ integers. In this case the voltage has a period $P$ times longer then the period of the main trapping RF. In dimensionless units the period of the function $f(\xi)$ is equal to $T=P \pi$. With the condition $f(\xi)=f(\xi+T)$, eq 4 is a linear differential equation with periodic coefficients, the Hill equation [8,9]. Analytical methods for the solution of the Hill equation have been developed [9]. Matrix methods are reviewed here.

\section{Matrix Method in the Theory of Equations with Periodic Factors}

\section{Matrix Methods and the Stability Condition}

The method applied here to investigate the stability of the solutions of the Hill equation is described in detail in [10]. It is based on two fundamental characteristics of eq 4: Linearity and periodicity. Consider the pair of independent solutions $u_{1}(\xi)$ and $u_{2}(\xi)$ of eq 4 through one single RF period $(0<\xi<T)$. Define these solutions by the initial conditions

$$
u_{1}(0)=1 ; \quad u_{1}^{\prime}(0)=0 ; \quad u_{2}(0)=0 ; \quad u_{2}^{\prime}(0)=1 \text {. }
$$

where $u_{1}{ }^{\prime}(\xi)=d u_{1} / d \xi$ and $u_{2}{ }^{\prime}(\xi)=d u_{2} / d \xi$.

Because eq 4 is linear, the general solution with initial conditions $u(0)=x_{0}$ and $u^{\prime}(0)=v_{0}$ can be expressed as a linear superposition of the two independent particular solutions as follows: 


$$
u(\xi)=x_{0} u_{1}(\xi)+v_{0} u_{2}(\xi)
$$

Because the solutions $u_{1}(\xi)$ and $u_{2}(\xi)$ may be calculated for any time $\xi$, eq 6 is valid for any time. However, as shown below, we need to calculate these solutions through only one RF period. Because of the periodicity of eq 4 , the same solutions $u_{1}(\tau)$ and $u_{2}(\tau)$ will be valid for $\tau=\xi-T$ during the second period $T<\xi<2 T$. In order to express the solution with the same initial conditions at $\xi=0$, consider the solution at $\xi=T$. From eq 6 one derives

$$
\begin{aligned}
& u(T)=x_{0} u_{1}(T)+v_{0} u_{2}(T)=x_{1}, \\
& u^{\prime}(T)=x_{0} u_{1}^{\prime}(T)+v_{0} u_{2}^{\prime}(T)=v_{1} .
\end{aligned}
$$

The solution during the second period is expressed as

$$
u(\xi)=x_{1} u_{1}(\tau)+v_{1} u_{2}(\tau), \quad \tau=\xi-T
$$

With the same considerations, the solution after the ( $n$ $+1)$ th period can be expressed as follows

$$
u(\xi)=x_{n} \cdot u_{1}(\tau)+v_{n} \cdot u_{2}(\tau), \quad \tau=\xi-n T
$$

The representation of the solution as in eq 9 is called a state vector representation [11]. With eq 9 it is possible to calculate the trajectory of an ion at any time. In order to do this we need to (1) calculate the pair of independent solutions $u_{1}(\tau)$ and $u_{2}(\tau)$ through one period $0<\tau<T$ and (2) calculate the sequence of state vectors $\left(x_{n}, v_{n}\right)$. Eq 9 can be written in matrix form as

$$
\begin{aligned}
& \left(\begin{array}{c}
x_{n+1} \\
v_{n+1}
\end{array}\right)=\mathrm{M} \cdot\left(\begin{array}{l}
x_{n} \\
v_{n}
\end{array}\right)=\mathrm{M}^{n} \cdot\left(\begin{array}{l}
x_{0} \\
v_{0}
\end{array}\right), \\
& \text { where }\left[\begin{array}{cc}
u_{1}(T) & u_{2}(T) \\
u_{1}^{\prime}(T) & u_{2}^{\prime}(T)
\end{array}\right]=\left[\begin{array}{ll}
m_{11} & m_{12} \\
m_{21} & m_{22}
\end{array}\right] .
\end{aligned}
$$

Methods to calculate the $n$th power of a $2 * 2$ matrix are described in [12] and in Appendix 1 and give:

$$
\begin{aligned}
& \mathrm{M}^{n}= \\
& {\left[\begin{array}{cc}
\cos (n T \beta)+A \sin (n T \beta) & \mathrm{B} \sin (n T \beta) \\
-\Gamma \sin (n T \beta) & \cos (n T \beta)-A \sin (n T \beta)^{(1]}
\end{array}\right)} \\
& A=\frac{m_{11}-m_{22}}{2 \cos (T \beta)}, \quad B=\frac{m_{12}}{\sin (T \beta)}, \quad \Gamma=\frac{-m_{21}}{\sin (T \beta)}
\end{aligned}
$$

Here $\beta$ is a stability number, which can be calculated from the equation

$$
2 \cos (\pi \beta)=m_{11}+m_{22}
$$

Note that $m_{11}+m_{22}$ is the trace of the matrix.

In the mathematical literature the $\mathrm{M}$ matrix is usually called the monodromy matrix $[9,12 \mathrm{c}]$, in the engineering literature it is called the transmission matrix [11], and in ion optics it is called the transfer matrix [12b]. It determines the global stability of the solutions of an equation with periodic coefficients. For stable solutions it is necessary that $\left|m_{11}+m_{22}\right|<2$. In this case $\beta$ is a real number which can be calculated from eq 12 with unit uncertainty: If $\beta$ is a solution of eq 12 , then each number $\beta \pm N$ will also be a solution. In mass spectrometry the number $\beta$ is called the stability parameter [3]. The space of trapping voltage parameters is divided into an infinite number of stable and unstable regions depending on the value of $\beta$. For motion in a single direction the stable regions can be numbered in increasing order $N=$ $1,2,3, \ldots$. Inside each stable region eq 12 has roots from 0 to 1 . Because of the uncertainty in the definition of $\beta$, it is useful to accept $N-1<\beta<N$ inside the $N$ th stable region.

If $\left|m_{11}+m_{22}\right|>2$ the motion is unstable. In this case the solutions of eq 12 for $\beta$ are imaginary. It is useful to consider $\mu$, the increment of exponential growth of the ion oscillation amplitude during one period. It is given by

$$
2 \cosh (\mu)=\left|m_{11}+m_{22}\right|
$$

The calculation of the general solution of the Hill equation is thus reduced to the calculation of two independent solutions of the equation through one period. This may be done by any suitable numerical method. For the special case of rectangular waveforms, the solutions can be obtained analytically. The next section describes a matrix method for the numerical calculation of the pair of solutions of eq 4 .

\section{A Matrix Method to Calculate Independent Solutions of the Hill Equation}

From the calculation of the two independent pair of solutions one derives the values of matrix

$$
\mathrm{U}(\tau)=\left[\begin{array}{cc}
u_{1}(\tau) & u_{2}(\tau) \\
u_{1}{ }^{\prime}(\tau) & u_{2}{ }^{\prime}(\tau)
\end{array}\right]
$$

at each moment $\tau$ inside the first period $[0, T]$. In particular, the transfer matrix $M$ is equal to $U(T)$. The calculation of this matrix must be as accurate as possible. The matrix $\mathrm{M}$ has a special property. The determinant of the matrix is unity, i.e., $\operatorname{det}[\mathrm{M}]=1$, because at any time we have $\operatorname{det}[\mathrm{U}(\tau)]=1$. This property is a consequence of the general Liouville theorem [12b, 13] which requires that the area in phase space of a beam of ions be conserved. In any numerical solution of the equation of motion (eq 4) one must carefully check that this condition is fulfilled.

To solve the equation numerically we approximate 
the varying voltage with a constant value over short times. In this approach the full period of the function $f(\xi)$ is divided into a finite number of elements, $K$, and the voltage is taken to be constant during each element [10]. For each element the solution of the equation is calculated exactly and analytically and the solution over the entire period is then calculated consecutively. The condition of the Liouville theorem, $\operatorname{det}[\mathrm{U}(\tau)]=1$, is strictly valid for each step, because the solution is calculated from exact analytical expressions.

The method is described in references [10-12a]. The period $[0, T]$ is divided into a number of intervals at points $\tau_{k}, k=1,2, \ldots K-1$. Consider the $k$ th interval. The voltage is equal to $f_{k}=f\left(\tau_{k}\right)$, and the duration is $\Delta_{k}=\tau_{k}$ $-\tau_{k+1}$. The particle position and velocity at the beginning of the interval are $x_{k}$ and $v_{k}$. For the case of a constant voltage $f(\tau)=f_{k}$, eq 4 can be solved analytically:

$$
\begin{aligned}
u(\tau) & =x_{k} \cos \left(\tau-\tau_{k}\right) \sqrt{f_{k}} \\
& +\frac{v_{k}}{\sqrt{f_{k}}} \sin \left(\tau-\tau_{k}\right) \sqrt{f_{k}}, \text { if } f_{k}>0,
\end{aligned}
$$

or

$$
\begin{aligned}
& u(\tau)=x_{k} \operatorname{Cosh}\left(\tau-\tau_{k}\right) \sqrt{-f_{k}}+\frac{v_{k}}{\sqrt{-f_{k}}} \operatorname{Sinh}(\tau \\
& \left.-\tau_{k}\right) \sqrt{-f_{k}} \text { if } f_{k}<0 .
\end{aligned}
$$

The position $x_{k+1}=u\left(\tau_{k+1}\right)$ and velocity $v_{k+1}=$ $u^{\prime}\left(\tau_{k+1}\right)$ of a particle at the beginning of the next interval is calculated from eq 15. Again, this can be written as a matrix equation:

$$
\left(\begin{array}{c}
x_{k+1} \\
v_{k+1}
\end{array}\right)=\mathrm{V}\left(f_{k}, \Delta_{k}\right) \times\left(\begin{array}{c}
x_{k} \\
v_{k}
\end{array}\right)
$$

where

$\mathrm{V}(f, \Delta)=\left[\begin{array}{cc}\operatorname{Cos} \Delta \sqrt{f} & \frac{1}{\sqrt{f}} \operatorname{Sin} \Delta \sqrt{f} \\ -\sqrt{f} \operatorname{Sin} \Delta \sqrt{f} & \operatorname{Cos} \Delta \sqrt{f}\end{array}\right], \quad$ if $f>0$,

or

$$
\begin{aligned}
& \mathrm{V}(f, \Delta)= \\
& {\left[\begin{array}{cc}
\operatorname{Cosh} \Delta \sqrt{-f} & \frac{1}{\sqrt{-f}} \operatorname{Sinh} \Delta \sqrt{-f} \\
\sqrt{-f} \operatorname{Sinh} \Delta \sqrt{-f} & \operatorname{Cosh} \Delta \sqrt{-f}
\end{array}\right], \quad \text { if } f<0 .}
\end{aligned}
$$

With the use of eq 16 the solution can be calculated at any desired time directly from the initial conditions $x_{0}$ and $v_{0}$. In order to calculate the pair of independent solutions with the initial conditions of eq 5 , we need to do the calculation twice. It is useful to combine these two calculations in one matrix equation. According to eq 5 the initial condition for the $U$ matrix at $\tau=0$ is $\mathrm{U}(0)=1$. It follows from eq 16 that

$$
\begin{aligned}
\mathrm{U}\left(\tau_{k}\right)= & \mathrm{V}\left(f_{k}, \Delta_{k}\right) \times \mathrm{V}\left(f_{k-1}, \Delta_{k-1}\right) \times \ldots \\
& \times \mathrm{V}\left(f_{1}, \Delta_{1}\right) .
\end{aligned}
$$

Eq 18 gives the matrix of fundamental solutions $U$ at any desired moment. It is the consecutive product of transmission matrices of all preceding intervals. In particular, the transfer matrix for one period (the monodromy matrix) is the product of the matrices of all intervals over one cycle i.e.,

$$
\begin{aligned}
\mathrm{M} & =\mathrm{U}\left(\tau_{K}\right)=\mathrm{V}\left(f_{K}, \Delta_{K}\right) \\
& \times \mathrm{V}\left(f_{K-1}, \Delta_{K-1}\right) \times \ldots \times \mathrm{V}\left(f_{1}, \Delta_{1}\right)
\end{aligned}
$$

This method is simple, general and the condition of the Liouville theorem $(\operatorname{det}[\mathrm{M}]=1)$ is valid automatically. The method is approximate because it represents the smooth function $f(\xi)$ by a series of steps. The error in calculating $\beta$ is proportional to $h$, where $h=p / K$, the interval width.

Conventional methods of calculating the stability of ion motion and thus the stability diagram $[2,4]$ require the numerical solution of a nonlinear algebraic equation, and this requires the calculation of infinite fractions. A complicated procedure for distinguishing between the infinite number of roots is needed. In the case of the Hill equation, the stability parameter $\beta$ is defined as an eigenvalue of an infinite matrix [2]. The calculation of eigenvalues is one of the most complicated procedures in computational mathematics. The time required for the calculation increases exponentially with the size of the matrix. In comparison, the matrix method described here gives answers in one simple calculation. There is only one limit to the matrix method described here: The smooth voltage function $V(t)$ is represented by a series of steps. However, this concerns only the method as it is used in this paper. The accuracy of the method may be improved easily by the use of any good program for the numerical solution of the Hill equation over one period with the actual smooth voltage function. In all the applications which are discussed below, the approximate method used here gives sufficient accuracy, provided the time step is small.

\section{Examples of Stability Diagram Calculations}

\section{Stability Diagrams with Rectangular Waveforms}

A square wave voltage is the simplest case. This was first considered by Richards et al. [7] who calculated the first stability region for a rectangular wave with different periods for the positive and negative parts of the 
waveform over one cycle. Here we extend the calculation to higher stability regions and consider other waveforms. The time dependence of the voltage in this case can be written as

$$
\begin{aligned}
V(t) & =\left\{\begin{array}{c}
U_{1}, \text { for } \_0<<t_{1} \\
U_{2}, \text { for } t_{1}<t<T_{R F}
\end{array}, \quad V\left(T_{R F}+t\right)\right. \\
& =V(t), T_{R F}=\frac{2 \pi}{\Omega} .
\end{aligned}
$$

It is appropriate to use dimensionless units

$$
\begin{aligned}
f_{1,2} & =\frac{8 e U_{1,2}}{m \Omega^{2} r_{0}^{2}}, \quad d=\frac{t_{1}}{T_{R F}}, \quad \Delta_{1}=\pi d, \\
\Delta_{2} & =\pi(1-d) .
\end{aligned}
$$

This choice of parameters follows from averaging of the ion motion for the case of a pulsed voltage waveform, as described in [14]. It is useful to present the stability diagram on the plane of parameters

$$
a=d f_{1}+(1-d) f_{2}, \quad q=\left(f_{1}-f_{2}\right) d(1-d) .
$$

For the simplest case of a symmetric pulsed voltage, $q$ is equal to a quarter of the $f(\xi)$ min-max difference (Figure 1a). The parameter $a$ equals the average DC component of the time dependent voltage. A comparison of the conventional Mathieu stability diagram and the diagram with a pulsed voltage waveform is given in [14].

With a fixed value of $f_{1} / f_{2}$ it is possible to calculate analytically the boundaries of the stable regions in the plane of pulse parameters $\left(f_{1} \Delta_{1}, f_{2} \Delta_{2}\right)$ [15]. Here we use the more general matrix methods. From eq 19 the transmission matrix is calculated as follows:

$$
\mathrm{M}=\mathrm{V}\left(a-\frac{q}{(1-d)^{\prime}}, \Delta_{2}\right) \cdot \mathrm{V}\left(a+\frac{q}{d^{\prime}} \Delta_{1}\right) .
$$

It is possible to calculate the transmission matrix from eq 23 for any given values of the parameters $a$ and $q$. Depending on the value of $s(a, q)=0.5\left|m_{11}+m_{22}\right|$, one can find the stability parameter $\beta$ from eq 12 , or the increment $\mu$ from eq 13. In order to determine the stability diagram we need only to define the stability or instability of the ion motion. Each point on the plane of parameters is marked with a black color where it corresponds to stable ion motion, and with white in the opposite case. The resulting stability diagram is shown in Figure 1b. The higher regions of stability are shown here for the first time.

Figure $1 \mathrm{~b}$ shows the stability diagram with a nonharmonic voltage waveform. The Fourier spectrum of a periodic rectangular wave consists of an infinite number of overtones of the main trapping frequency $\Omega$ with frequencies $\Omega_{n}=n \Omega, n=0,1,2, \ldots$. Each of these overtones causes a series of parametric resonances (see (a)

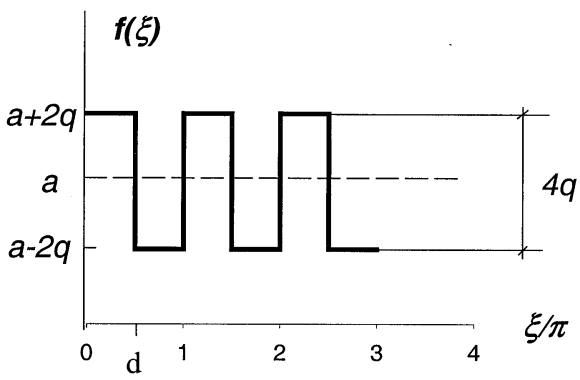

(b)

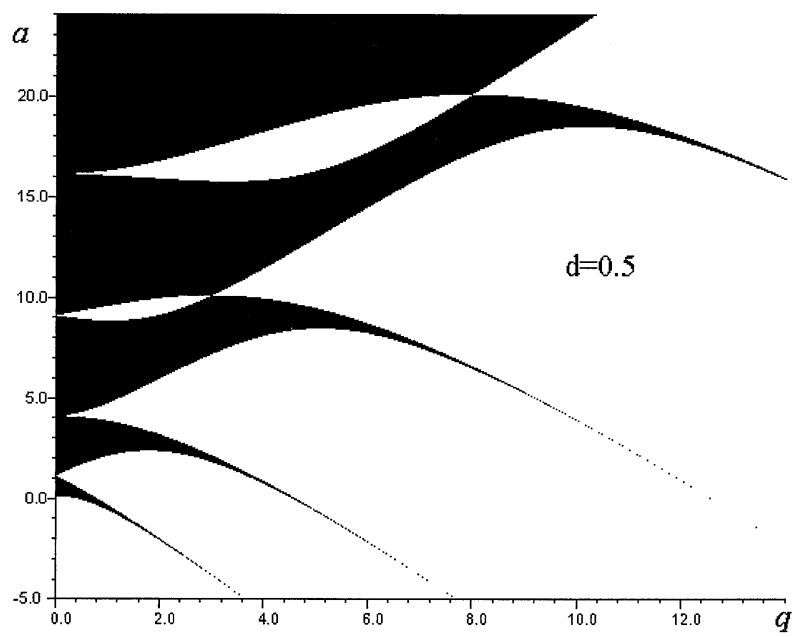

Figure 1. Stability diagram with a pulsed trapping voltage of the simplest form. (a) The form of the dimensionless $\pi$-periodic function $f(\xi)$ and definition of the dimensionless parameters $a$ and q. (b) Stability diagram for the Hill equation (eq 4) solutions. Black regions have stable motion.

below). For the overtone with frequency $\Omega_{n}$, parametric resonance bands start from the $a$-axis at the positions $a=\left(0.5 K \Omega_{n}\right)^{2}=K^{2} n^{2}$. Here we use dimensionless units, so $\Omega_{n}=2 n$, and $K=1,2,3 \ldots$. is the order of resonance. Hence, the parametric resonance band, which starts from $a=1$ is governed by the first order resonance at the main frequency $\Omega_{1}$. The second band, which starts from $a=4$, is governed by the second order resonance $(K=2)$ at the main frequency $\Omega_{1}(n=1)$, and first order resonance $(K=1)$ at the second overtone $\Omega_{2}(n=2)$, and so on. In the particular case of a symmetric square wave as in Figure 1a, the amplitude of the second overtone is zero due to symmetry, and the waveform does not contain a second overtone. The first and second parametric bands in Figure $1 \mathrm{~b}$ do not have any singularities, because they are governed by the resonance at the lowest frequency $\Omega_{1}=\Omega$. The third band is governed by the third order resonance at $\Omega_{1}$ and the first order resonance at $\Omega_{3}=3 \Omega$. The width of a resonance band depends on the amplitude of the excitation harmonic. The complicated behavior of the width of the third resonance band in Figure $1 \mathrm{~b}$ is explained by the combined influence of two harmonics, with well defined amplitudes and phases. Even more complicated behavior is seen in higher regions of instability of Figure $1 b$. 
(a)

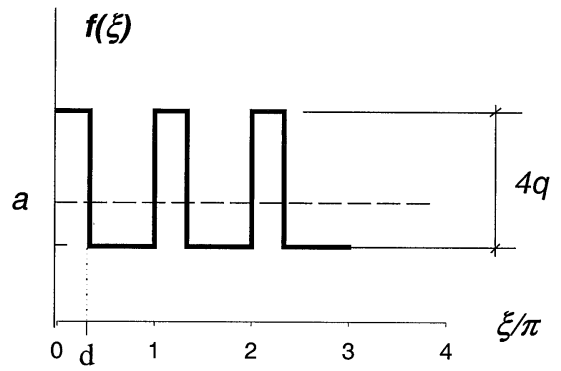

(b)

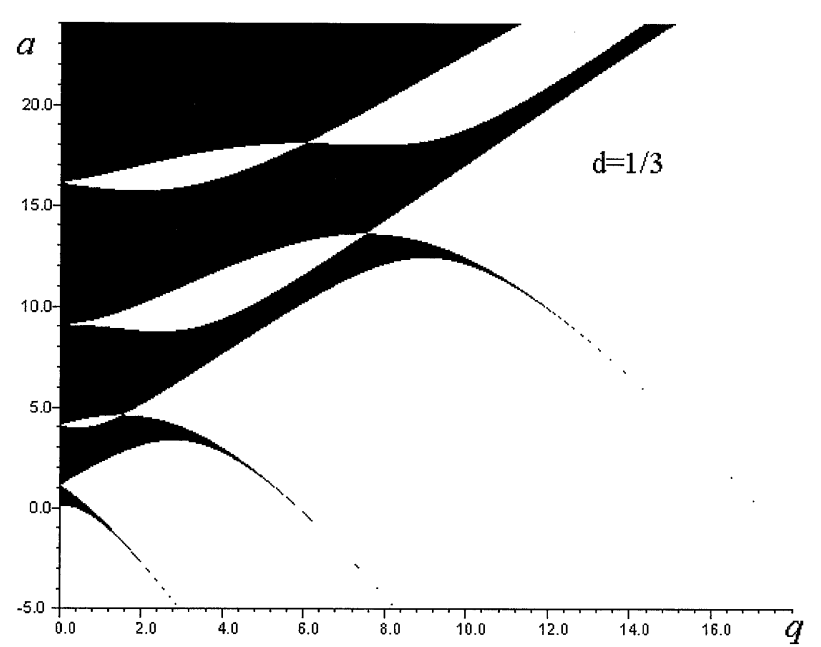

Figure 2. Stability diagram with a pulsed waveform of duty cycle $d=1 / 3$. (a) The waveform and definition of the parameters $a$ and $q$. (b) Stability diagram for the Hill equation (eq 4) solutions. Black regions have stable motion.

This can be called an interference of parametric excitations. In the case of a pulsed waveform of general asymmetric shape, which has $\Delta_{1} \neq \Delta_{2}$ (Figure 2a), the amplitude of the second overtone is not equal to zero. The second unstable band has a first order resonance at $\Omega_{2}$ and a second order resonance at $\Omega_{1}$. Interference phenomena are present in the second unstable band of Figure 2b.

As a third and new example of pulsed voltages, consider the voltage waveform of Figure 3a. The transmission matrix is calculated as

$$
\begin{aligned}
\mathrm{M} & =\mathrm{V}(a, \pi-\Delta) \cdot \mathrm{V}(a-2 q, 0.5 \Delta) \cdot \mathrm{V}(a \\
& +2 q, 0.5 \Delta), \Delta=\pi \frac{t_{1}}{T}
\end{aligned}
$$

The stability diagram for the case $\Delta=0.5 \pi$, is shown in Figure $3 \mathrm{~b}$. Each overtone causes a first order parametric resonance at small $q$ when $a$ is close to the resonance points $a=K^{2}$. Thus the width of each unstable area in the region of small $q$ values is linearly proportional to $q$. There is no such point inside higher unstable bands, where the width of the band equals zero. Interference of overtones leads to complex behavior of the boundaries of stable regions.

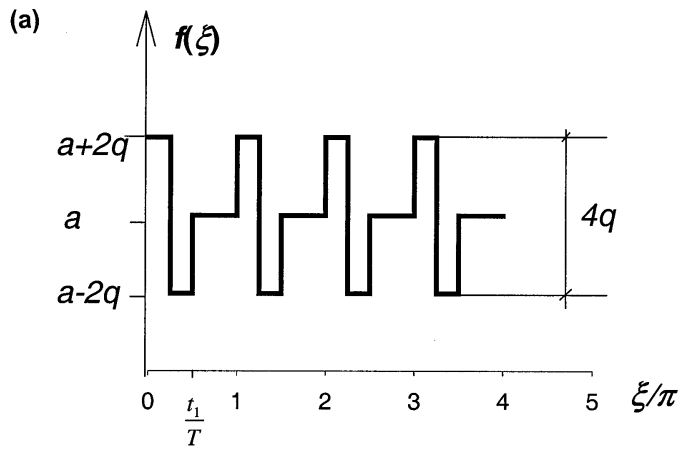

(b)

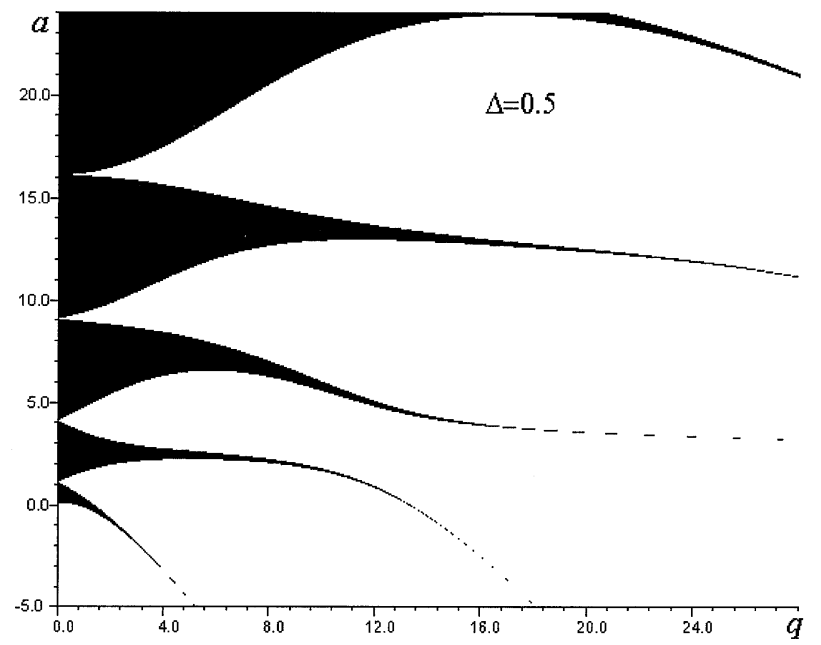

Figure 3. Stability diagram with a pulsed bipolar waveform. (a) The waveform and definition of the parameters $a$ and $q$. (b) Stability diagram for the Hill equation (eq 4) solutions. Black regions have stable motion.

\section{Ince Diagram for the Mathieu Equation}

For the usual case of an RF waveform, the trapping voltage is given by $V(t)=U-V_{\mathrm{RF}} \operatorname{Cos}(\Omega t)$. The corresponding dimensionless function $f(\xi)$ in eq 4 is:

$$
\begin{aligned}
f(\xi) & =a-2 q \operatorname{Cos}(2 \xi), \quad a=\frac{8 e Z_{i}}{M r_{0}^{2} \Omega^{2}} \\
U, q & =\frac{4 e Z_{i}}{M r_{0}^{2} \Omega^{2}} V_{R F} .
\end{aligned}
$$

In this case eq 4 is the Mathieu equation. The stability diagram for solutions of this equation was calculated and shown for the first time by Ince [1]. Here we describe the matrix method for the calculation of the diagram. In order to calculate the transmission matrix the entire period is divided into a number of intervals during which the voltage is assumed to be constant [12]. In our experience, dividing the period into 1000 equal intervals leads to at least 4 digits of accuracy in the calculation of the stability parameter. This is not sufficient if higher accuracy is needed, for example for ion trap experiments with very high resolution [16]. How- 
(a)

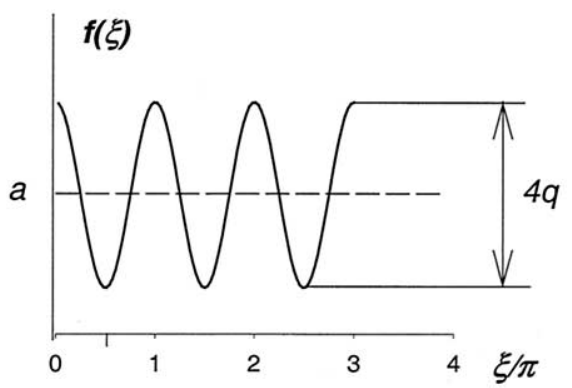

(b)

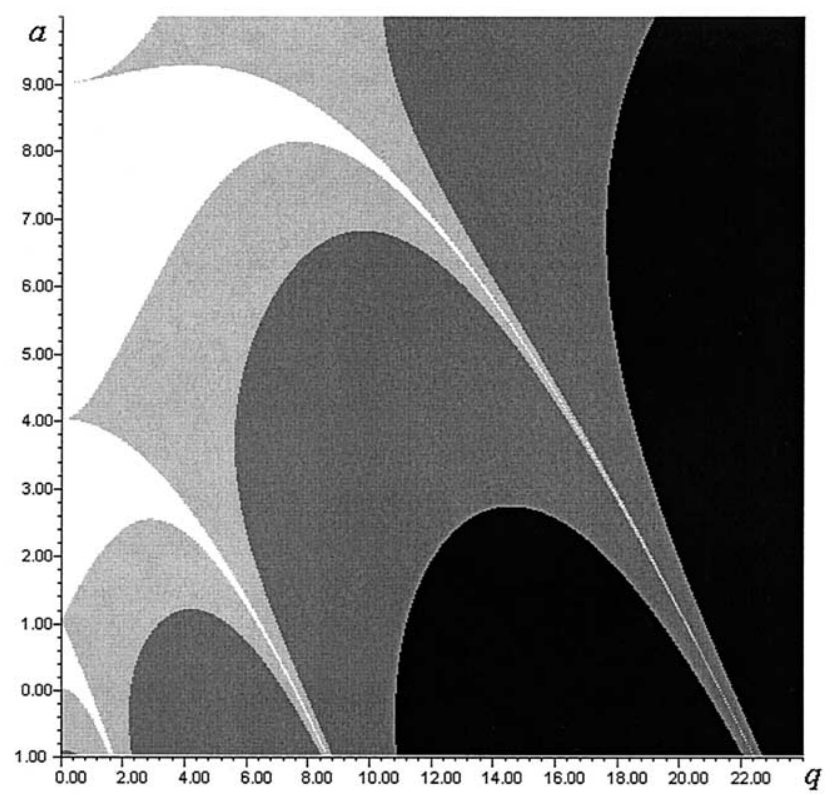

Figure 4. The map of stability for the Mathieu equation. (a) The waveform and definition of the parameters $a$ and $q$. (b) Stability diagram. White regions have stable motion. Unstable regions are split into a number of bands according to the value of exponential increment. Boundaries of regions are the lines of equal $\mu$. Light gray areas have $0<\mu<2.8$, medium gray $2.8<\mu<7.0$, and black $\mu>7.0$.

ever, this accuracy is sufficient for calculation of the stability diagram. In practice, there are many different factors that influence the ion oscillation frequency [17] and which can lead to shifts and shape perturbations of the stability diagram. The nature of these factors sometimes is unknown [18].

In the case of the Mathieu equation, we use the method described above. In order to derive information about the comparative stability of different points on the plane of voltage parameters, it is advantageous to use different shades depending on the value of the increment in the unstable regions. This is shown in Figure 4. At a resolution of $600 * 400$ pixels and 500 intervals in one RF period, such a calculation takes $0.5 \mathrm{~h}$ with a Pentium computer (166 MHz). This is quite reasonable, considering that we need to calculate this diagram only once. The speed of the calculation may be increased if the array of $s(a, q)$ is calculated over a coarser mesh and used later with interpolation.

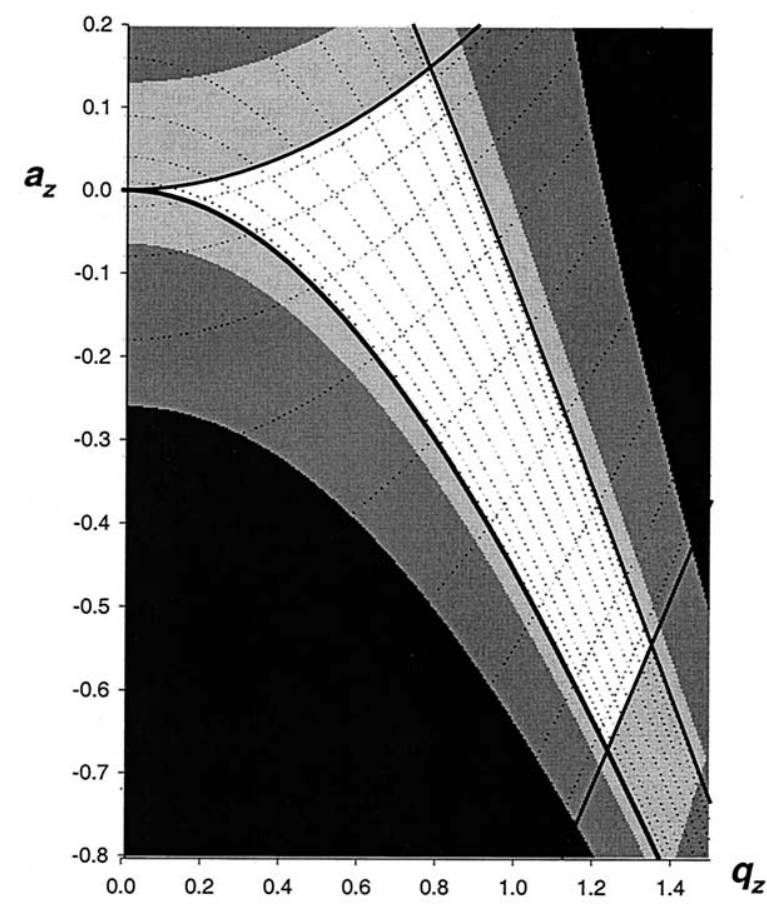

Figure 5. First region of stability of a 3-D trap. The white area has stable motion. Solid lines are the boundaries $\beta=1$ and $\beta=0$ of the first stable zone for axial and radial motions. Dotted lines are iso- $\beta$ lines. Regions in the unstable area are shaded according to the value of $\mu=\max \left(\mu_{z}, \mu_{r}\right)$ as follows: gray $0<\mu<0.8$, medium gray $0.8<\mu<2.1$, and black $\mu>2.1$.

\section{The Combined Stability Diagram for a Trap and for a Mass Filter}

The stability diagrams in Figures $1-4$ are for motion in only one direction, $z$-motion in a trap, or $x$-motion in a mass filter. Ion motion in a quadrupole field occurs in three independent directions. The parameters of motion for each are different. For example, the Mathieu parameters for a trap are $a_{x}=a_{y}=-0.5 a_{z}, q_{x}=q_{y}=-0.5 q_{z}$. The sign of $q$ does not influence the stability of ion motion, because a change of sign is equivalent to an RF-voltage phase shift and the stability diagram is symmetric about the $a$ axis $(q=0)$. Thus the stability diagram is usually shown for positive $q$. Stable 3-D motion occurs for the points where motion in all three independent directions is stable simultaneously. In order to present the general diagram, the transmission matrix can be calculated for the equation of motion for each direction. The ion motion will be stable in both $x$ and $z$ if the maximum of the numbers $s_{x}$, or $s_{z}$ is less than unity. In order to plot the general diagram we use the same method as with the Ince diagram (previous section), but with the use of the number $s=\max \left(s_{z}, s_{x}\right)$. This reproduces the well known first region of stability of a 3-D trap on the plane $\left(a_{z}, q_{z}\right)$ as is shown in Figure 5.

In a mass filter the quadrupole field influences the ion motion transverse to the $z$ axis only, and $a_{x}=-a_{y}$ $q_{x}=-q_{y}$. Figure 6 shows the calculated boundaries of stable regions for a wide range of $a_{x}$ and $q_{x}$. The bound- 


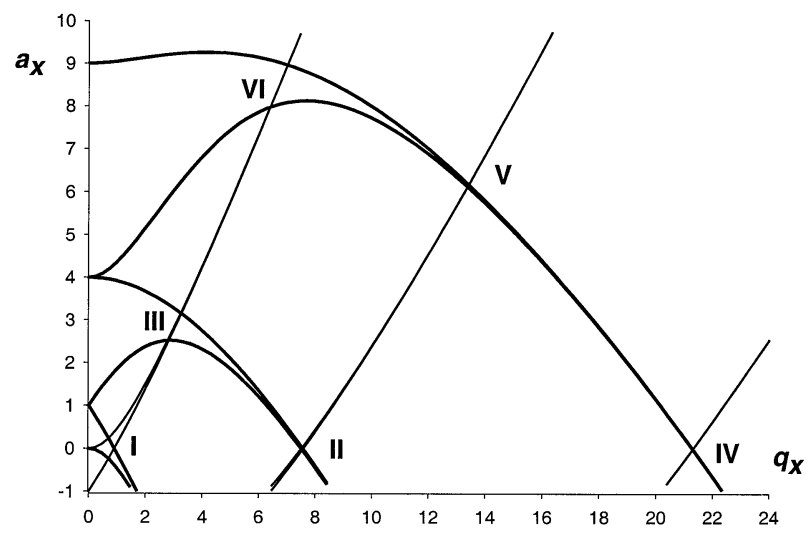

Figure 6. General stability diagram for the ion motion in a 2-D quadrupole field. The boundaries of stable regions are shown with solid lines. Numbers indicate different regions of simultaneous stability of $x$ - and $y$-motion.

aries of stability are roots of the equation $|\mathrm{s}(a, q)|=1$ which was solved numerically. Figure 6 shows the notation for higher regions of stability, which will be used later in this article. (Others have used different notations.) The higher regions of stability are of interest because they offer high mass resolution or the ability to mass analyze ion beams of high kinetic energy (for example up to $\mathbf{R}_{1 / 2}=13,900$ at $m / z=39$ or unit resolution with ions of $10 \mathrm{keV}$ energy, in region IV [19]). In order to derive the diagram for combined stability in $x$ and $y$, we used the maximum of the numbers $s_{x}$ and $s_{y}$, as for the trap. The first (I), second (II), and fourth (IV) regions of stability calculated this way are shown in Figure 7. Results of the calculations for regions III, V, and VI are shown in Figure 8. Values of the $a$ and $q$ parameters at the tips of the stability diagrams are listed in Table 1 . For these calculations one RF period was divided into 5000 intervals. The trace of the monodromy matrix at the boundaries was 2.0 within an accuracy of $10^{-6}$. The $(a, q)$ values in Table 1 agree with values calculated from integer Mathieu functions to four significant digits.

\section{Stability Diagrams with Additional Quadrupole Excitation}

An auxiliary AC voltage of frequency $\omega$ which is different from the trapping frequency $\Omega$, is often used for resonant excitation of ion oscillations. Within the first stable region, the ion motion has the strongest Fourier harmonic at frequency $\omega_{s}=0.5^{*} \beta \Omega\left(\omega_{\mathrm{s}}\right.$ is the fundamental, secular frequency). A comparatively small auxiliary AC voltage can lead to significant changes in ion trajectories. Both dipole and quadrupole excitation are used in ion trap mass spectrometry. With dipole excitation, the electric field is independent of position. When the excitation frequency is close to the secular frequency $\omega_{\mathrm{s}}$, this leads to the usual resonance of a damped harmonic oscillator. In the absence of damping, the ion oscillation amplitude for excitation near the resonant frequency is finite and has a beat-like (a)

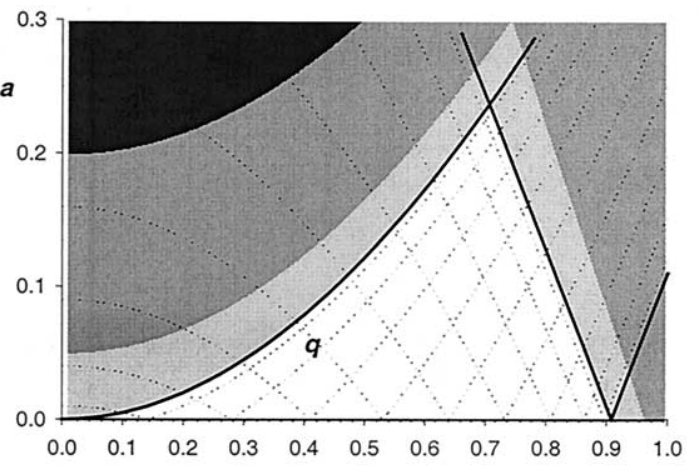

(b)

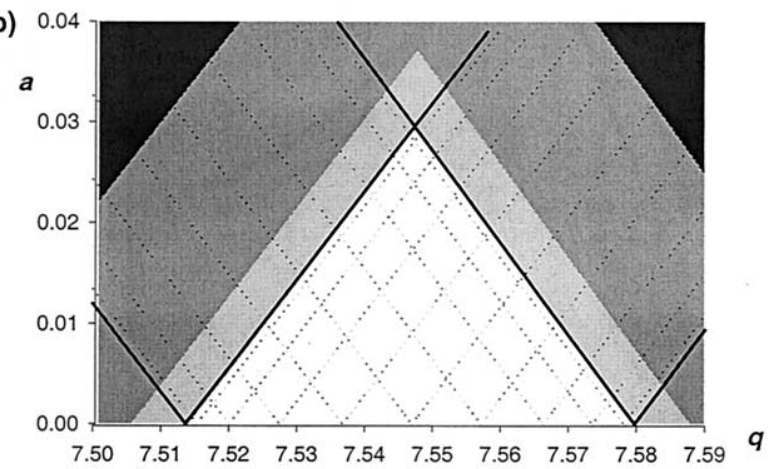

(c)

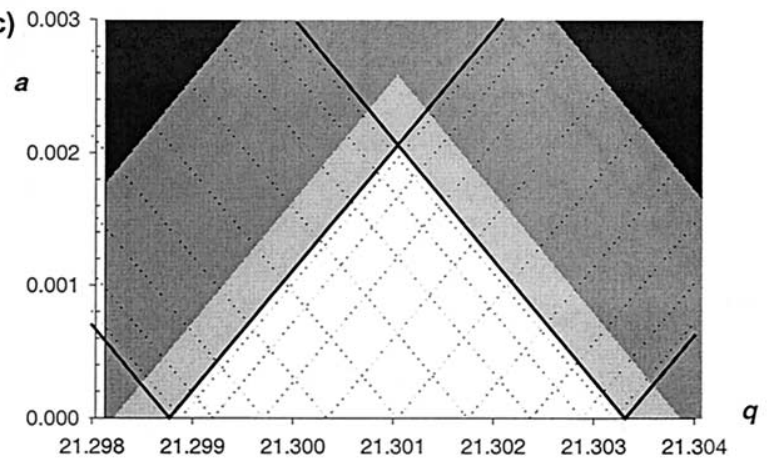

Figure 7. Regions of the general stability diagram for a mass filter: (a) First zone I; (b) second zone II; (c) fourth zone IV. These regions are symmetric about the line $a=0$, so the figure presents the upper part only. Solid lines are the boundaries of stability. Dotted lines are iso- $\beta$ lines. Regions in the unstable area are shaded according to the value of $\mu=\max \left(\mu_{x}, \mu_{y}\right)$ as follows: gray $0<\mu<0.5$, medium gray $0.5<\mu<1.4$, and black $\mu>1.4$.

structure. With quadrupole excitation the electric field is proportional to the distance from the center of the field. In this case the resonance has a parametric nature. Resonance appears at an infinite number of frequencies $[20,21]$. In particular, resonance at the secular frequency appears when the excitation frequency, $\omega_{K}$, is given by $\omega_{K}=2 \omega_{s / K}$, where $K=1,2, \ldots$ is the order of resonance. There is an entire interval of frequencies near each resonance value $\omega_{K}$, and the amplitude of oscillation increases exponentially if the excitation frequency is inside this interval. The width and position of the resonance band, and also the exponential increment, depend strongly on the auxiliary AC voltage amplitude. Linear viscous damping leads to a threshold for excitation $[12 c, 20]$. The auxiliary voltage must be greater than 
(a)

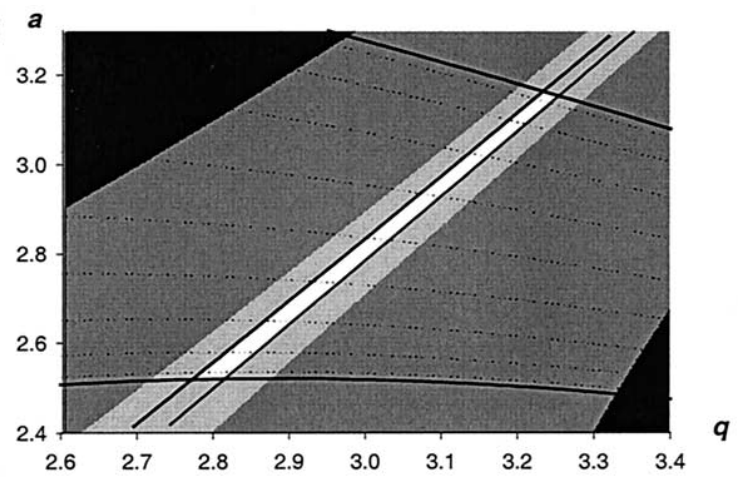

(b)

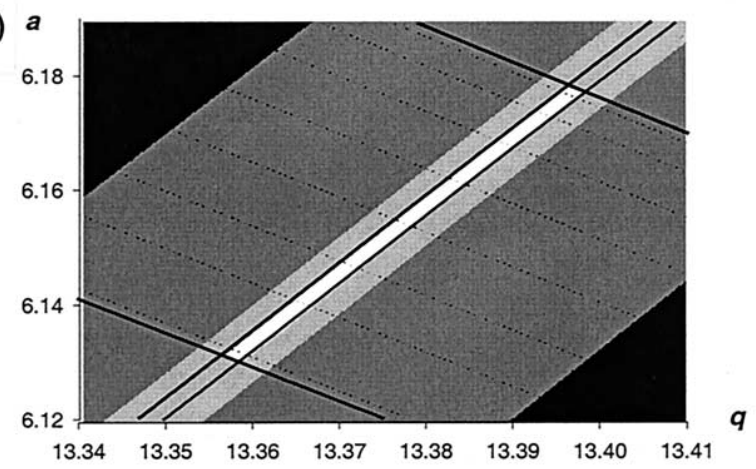

(c)

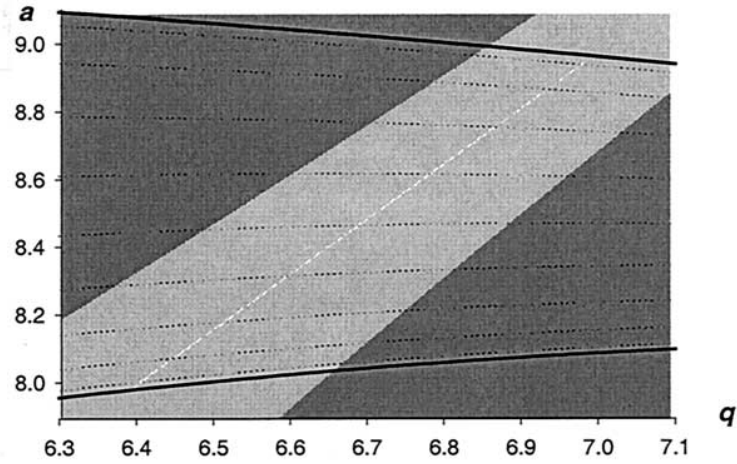

Figure 8. Additional regions of stability of a mass filter (a) zone III; (b) zone V; (c) zone VI. Solid lines are the boundaries of stability. Dotted lines are iso- $\beta$ lines. Boundaries of regions in unstable areas in (a) and (b) are: Gray $0<\mu<1.5$, medium gray $1.5<\mu<4.2$, and black $\mu>4$.2. Within the scaling of (c) the stable region VI is a thin line that passes through the $\mu<3$ region, shown in light gray. the threshold in order to observe parametric resonance. If the voltage is greater than the threshold, then the amplitude of ion oscillation increases exponentially, even with linear viscous damping. Unlike dipole excitation, resonant quadrupole excitation causes the ion oscillation amplitude to become infinite (in practice, ions strike the electrodes). The ion stability condition depends on the auxiliary quadrupole AC voltage and frequency.

The equation of ion motion in an RF quadrupole field with a second $\mathrm{AC}$ voltage, $-V_{\mathrm{AC}} \cos (\omega t)$ is

$$
u^{\prime \prime}+\left[a-2 q \cos 2 \xi-2 q^{\prime} \cos (2 \nu \xi+\alpha)\right] \cdot u=0 .
$$

Here the relative amplitude $q^{\prime}=4 e V_{\mathrm{AC}} / \mathrm{m} \Omega^{2} \mathrm{r}_{0}^{2}=\mathrm{q}$ $V_{\mathrm{AC}} / V_{\mathrm{RF}}, \nu=\omega / \Omega$ is the relative frequency, and $\alpha$ is the phase of the quadrupole excitation. If $\nu=N / P$, where $N$ and $P$ are integers, then the two harmonic functions in eq 26 have the same joint period $P \pi$. In this case eq 26 is periodic. The stability of the ion motion can then be investigated by the general theory of equations with periodic factors. To use this method it is necessary to calculate the solution matrix through the common period $P \pi$ and define the monodromy matrix $\mathrm{M}$ over the longer period. If the value $s\left(a, q, q^{\prime}, \nu\right)=0.5\left|m_{11}+m_{22}\right|$ is greater then unity, then the ion oscillations are unstable and the amplitude of ion oscillation increases exponentially. The increment over one period, $\mu$, is defined by

$$
\cosh (\mu P)=0.5 \cdot\left|m_{11}+m_{22}\right| .
$$

With a fixed excitation frequency, $\nu$, the stability diagram for eq 26 occupies a 3-D space $\left(a, q, q^{\prime}\right)$. It is useful to present results of the calculation for a fixed value of the excitation parameter $q^{\prime}$. This picture is a cross section at $q^{\prime}=$ constant , of the full stability diagram. Results of such a calculation for $\nu=1 / 5$ and several values of the excitation parameter, $q^{\prime}$, are shown in Figure 9. Figure 10 shows results of the calculation with $q^{\prime}=0.04$ (as in Figure 9c) but with several values of $\nu$

Table 1. The values of $(a, q)$ at the tips of the first six stability regions of a mass filter

\begin{tabular}{llllll}
\hline Region & Tip & $\beta_{x}$ & $\beta_{y}$ & $a_{x}$ & $\mathrm{q}_{x}$ \\
\hline \hline I & Upper & $1-$ & $0+$ & 0.236994 & 0.705996 \\
& Lower & $0+$ & $1-$ & -0.236994 & 0.705996 \\
II & Upper & $2-$ & $1+$ & 0.029550 & 7.5472754 \\
& Lower & $1+$ & $2-$ & -0.029550 & 7.5472754 \\
III & Upper & $2-$ & $0+$ & 3.164296 & 3.234075 \\
& Lower & $1+$ & $1-$ & 2.5209999 & 2.8153045 \\
IV & Upper & $3-$ & $2+$ & 0.0020608 & 21.300906 \\
& Lower & $2+$ & $3-$ & -0.0020608 & 21.300906 \\
V & Upper & $3-$ & $1+$ & 6.178418 & 13.396347 \\
& Lower & $2+$ & $2-$ & 6.130176 & 13.358318 \\
VI & Upper & $3-$ & $0+$ & 8.963711 & 6.993808 \\
& Lower & $2+$ & $1-$ & 7.976744 & 6.383490 \\
\hline
\end{tabular}


(a)

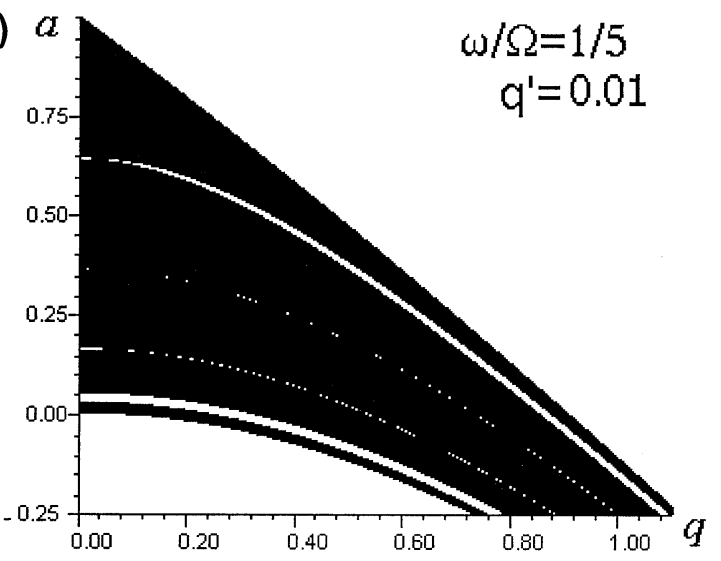

(b)
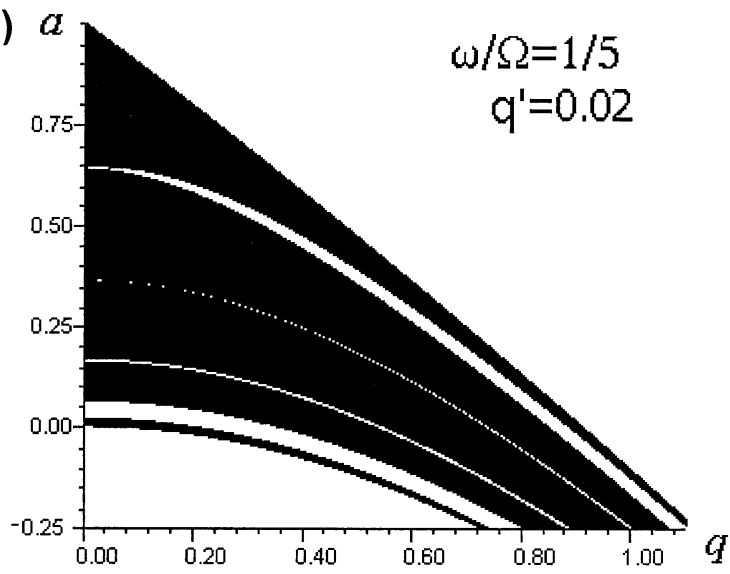

(c)

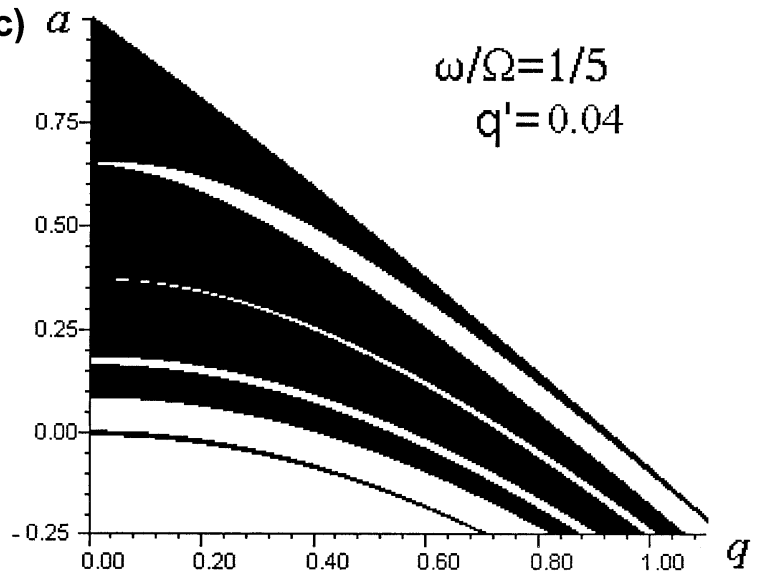

Figure 9. Stability diagram sections for the Mathieu equation with quadrupole excitation and by an auxiliary AC voltage of frequency $\omega=\Omega / 5$. The sections are calculated for a constant excitation parameter equal to (a) $q^{\prime}=0.01$; (b) $q^{\prime}=0.02$; (c) $q^{\prime}=$ 0.04. Black regions have stable motion.

with the same value of $P=10$. The values of $a$ and $q$ in Figure 9 and 10 are limited to the first stability region of the Mathieu equation. The auxiliary AC voltage leads to the appearance of lines of instability, which we call resonance lines. With a small excitation parameter, the resonance lines occur along iso- $\beta$ lines of the unperturbed diagram. The resonance values of $\beta$ are equal to (see Discussion): (a)

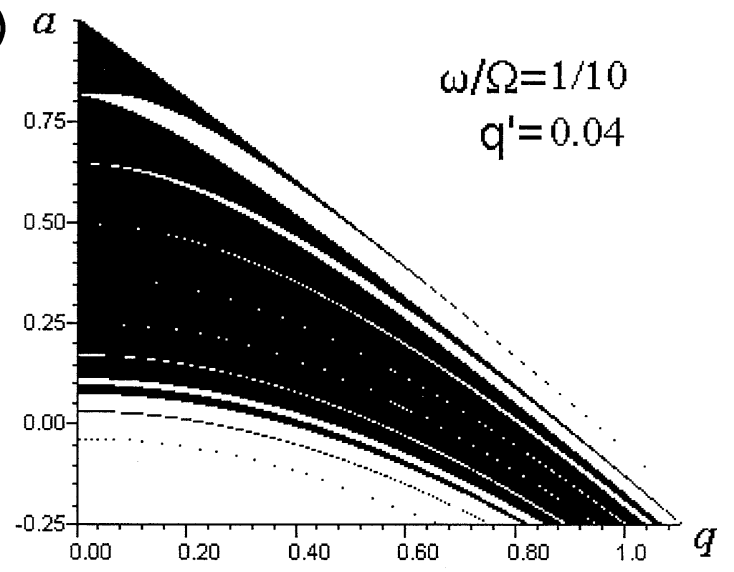

(b)

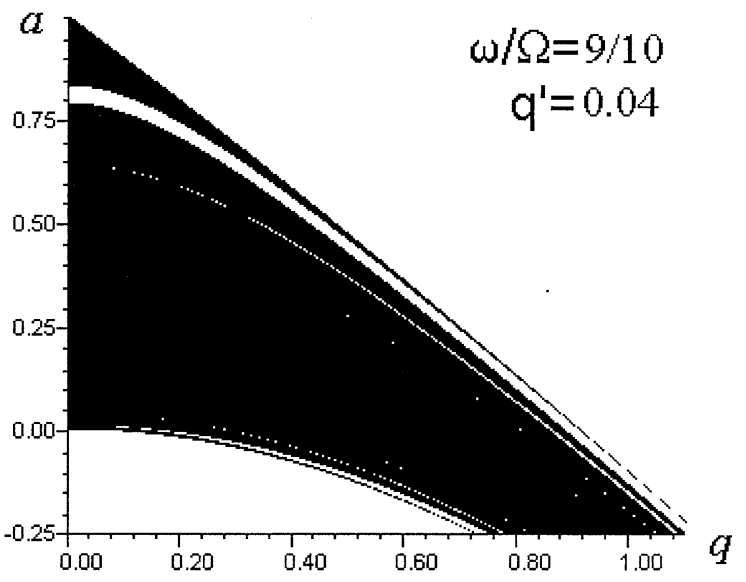

(c)

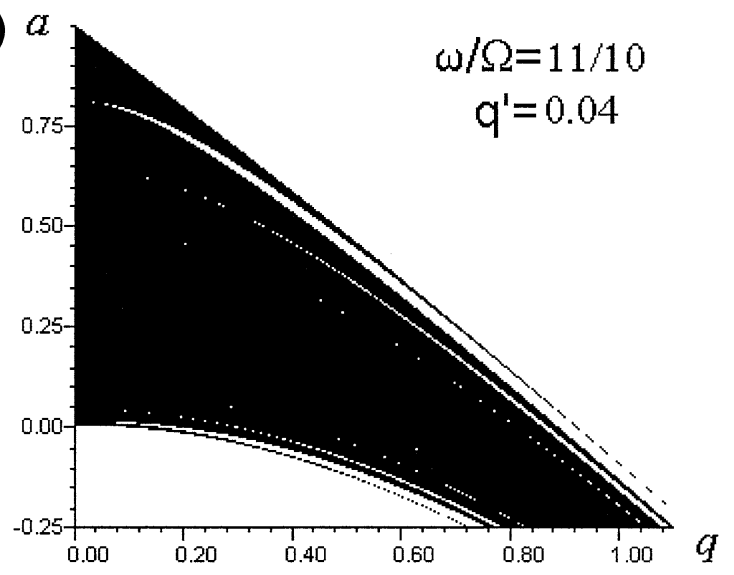

Figure 10. Stability diagram sections for the case of quadrupole excitation with excitation parameter $q^{\prime}=0.04$ and with frequency (a) $\omega=\Omega / 10$, (b) $\omega=9 \Omega / 10$, (c) $\omega=11 \Omega / 10$. Black regions have stable motion.

$$
\beta=k / P, k=1,2, \ldots P-1 .
$$

This equation follows from the general parametric resonance condition [21] for the case of a rational connection between the AC and RF frequencies, $\nu=$ $\omega / \Omega=N / P$. The number of resonance lines is equal to $P-1$. Hence, the first stability region splits into $P$ stable bands. 


\section{Combined Stability Diagram of a Mass Filter with Quadrupole Excitation}

Here we consider the combined stability diagram for both $x$ and $y$ motion with additional quadrupole excitation. Without quadrupole excitation the combined stability diagram of a mass filter is derived by overlapping the stable regions for $x$ and $y$ motion, with both expressed in terms of $a_{x}$ and $q_{x}$. (For the linear mass filter, the region of stability for $y$ motion in terms of $a_{x}$, $q_{x}$ is obtained by reflecting the diagram for $x$ motion in the $q_{x}$ axis). This diagram is useful because for a given rod size, operating frequency, DC and RF voltages, the $a$ and $q$ parameters are inversely proportional to ion mass. Ions with different mass are positioned along a scan line $a=\lambda q$ where $\lambda$ depends only on the ratio of DC to RF voltage: $\lambda=2 U / V$. The stability diagrams shown above were calculated with a fixed value of the excitation parameter $q^{\prime}$. The excitation parameter as well as trapping parameter $q$ depend on the ion mass. Ions with different masses have different excitation parameters $q$ '. Hence, the diagrams are not directly applicable to the analysis of the stability of ions of different masses in the same way as the Ince diagram.

With auxiliary quadrupole excitation the stability of ion motion depends on the four parameters $\left(a, q, q^{\prime}, \nu\right)$. It is difficult to imagine the stability diagram in four dimensions. With fixed values of $q^{\prime}$ and $\nu$ we can draw 2-D sections of the full four-dimensional diagram. Consider quadrupole mass filter operation near the tip of the first stability region. For a small mass range $\Delta M$ near some mass $M^{*}$, we can assume that the change in excitation parameter $q$ ' with ion mass is relatively small. In this case, the mass filter diagrams with instability lines caused by quadrupole excitation are directly applicable.

The calculated combined stability diagram near the tip of the first stable region of a mass filter with additional quadrupole excitation is shown in Figure 11. Here the quadrupole excitation frequency is a perfect fraction of the main trapping frequency, $\nu=9 / 10$. The boundaries of the normal stability diagram (without quadrupole excitation) are shown as solid black lines. The auxiliary quadrupole field leads to splitting of the tip into several islands of stability. The splitting is due to the appearance of several parametric resonance lines that are positioned along iso- $\beta$ lines. For the case $\nu=$ $9 / 10$ these are $\beta_{x, y}=1 / 10,2 / 10, \ldots, 8 / 10,9 / 10$. As the excitation parameter $q^{\prime}$ increases, the widths of the resonance lines increase and the stable islands move outside the boundaries of the normal stability diagram. That is, ion motion becomes stable in regions where it would be unstable without the auxiliary excitation. Results for other values of the quadrupole excitation frequency are similar.

The islands of stability can all be used in the usual manner for mass analysis. The most evident islands are labelled A, B, C, D in Figure 11. Ions with different mass to charge ratios have $a$ and $q$ parameters at $(a, q)$ (a)

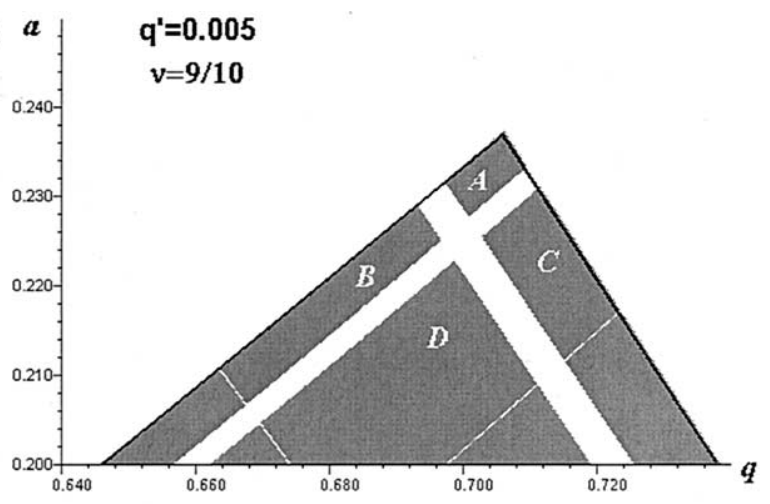

(b)

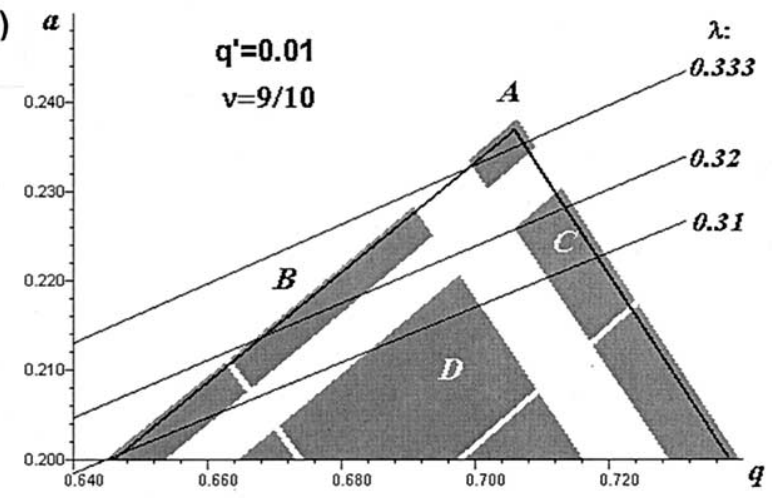

(c)

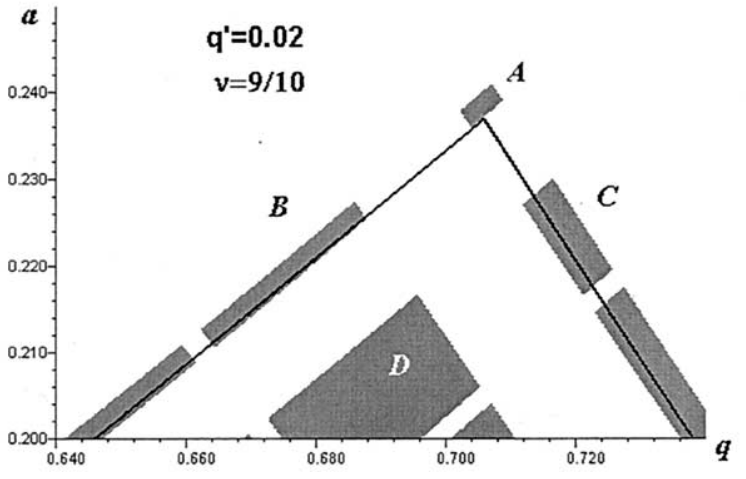

Figure 11. The combined stability diagram of a mass filter near the tip of the first stability zone with quadrupole excitation at frequency $\nu=9 / 10$. The calculation is performed with constant values of the excitation parameter $q^{\prime}$ (a) 0.005 , (b) 0.01 , (c) 0.02 . Grey regions give stable motion. The boundaries of the unperturbed diagram are shown with solid lines. Three scan lines with different ratios of $U / V$ are shown.

operating points positioned along a scan line $a=\lambda q$. The value of $\lambda$ defines the slope at which the scan line crosses the upper tip of the Mathieu diagram, and determines the range of ions of a particular $\mathrm{m} / \mathrm{z}$ that remain stable in the mass filter. Referring to Figure 11b, the scan line with slope $\lambda$ can cross island $\mathbf{A}$ only $(\lambda=$ $0.333)$, or two islands $\mathbf{B}$ and $\mathbf{C}(\lambda=0.320)$, or the three islands $\mathbf{B}, \mathbf{C}$, and $\mathbf{D}(\lambda=0.310)$, depending on $\lambda$ and the excitation parameter $q^{\prime}$. In these cases, singly, doubly, or triply overlapped mass spectra can be observed, respectively. Mass selection in islands $\mathbf{B}$ and $\mathbf{C}$ occurs with ion motion in different directions. In island $\mathbf{B}$ mass separation is achieved in the $y$ direction only, because $x$ 
motion is stable along the scan line at the left and right sides of island $\mathbf{B}$. Conversely, in island $\mathbf{C}$ mass separation occurs in the $x$ direction. The scan line crosses island $\mathbf{D}$ through the tip, hence mass separation in this island takes place as in the usual mass filter operation.

Mass separation in island $\mathbf{A}$ was used previously to modify and improve the peak shape [22]. The use of mass separation in the islands $\mathbf{B}, \mathbf{C}$, and $\mathbf{D}$ was recently tested experimentally with an ICP-MS system [23]. The experiment showed the effects of splitting of the stability diagram into a number of stable islands and the results were in good quantitative agreement with theory. Although the use of auxiliary quadrupole excitation does not give a significant increase in limiting resolution, the degradation of resolution and abundance sensitivity with increasing ion energy is considerably reduced. The peak tail of ${ }^{40} \mathrm{Ar}^{+}$at $\mathrm{m} / \mathrm{z}=39$ was eliminated and an abundance sensitivity of $10^{9}-10^{10}$ was achieved. For the ICP ion source, which has relatively large ion energy spread (up to several electron volts), this is very difficult to achieve by other means with operation in the first stability region. (An abundance sensitivity $>10^{9}$ with operation in the third region has been described [24].)

\section{RF-Only Quadrupole with Auxiliary Quadrupole Excitation}

The use of quadrupole excitation in a linear ion trap [25] provides an interesting case for theoretical and experimental discussion. Normally, the range $0<q<0.4$ of the first stability region is used for ion trapping. This simplifies the theoretical approach because the method of trajectory averaging (the pseudopotential approximation) is applicable. In this section we will mainly follow reference [26]. Consider the equations of ion radial motion in a 2-D quadrupole field with additional quadrupole excitation. These are eq 26 in which $a=a_{x}=-a_{y}, q=q_{x}=-q_{y}, q^{\prime}=q_{x}^{\prime}=-q_{y}^{\prime}$. Following Landau and Lifshitz [20a], substitute

$$
x=X+h_{x} \cos 2 \xi, \quad y=Y+h_{y} \cos 2 \xi .
$$

Here $X$ and $Y$ are relatively slow averaged radial motions, and $h_{x}$ and $h_{y}$ are amplitudes of fast oscillations at the frequency of the trapping RF. If trajectory averaging is applicable, then $h_{x}$ and $h_{y}$ follow the average motion and one can derive from eq 26 the following approximate expressions:

$$
h_{x}=\frac{q}{2} X, \quad h_{y}=-\frac{q}{2} Y .
$$

When these expressions are substituted back into eq 26 the equations for the averaged variables $X$ and $Y$ only are:

$$
\begin{aligned}
& \frac{d^{2} X}{d \xi^{2}}+\left[a+\frac{q^{2}}{2}\right] X=2 q^{\prime} \cos (2 \nu \xi+\alpha) X \\
& \frac{d^{2} Y}{d \xi^{2}}+\left[-a+\frac{q^{2}}{2}\right] Y=-2 q^{\prime} \cos (2 \nu \xi+\alpha) Y .
\end{aligned}
$$

After substitution of a new dimensionless time variable $\eta=\nu \xi$, eq 31 becomes Mathieu equations in canonical form:

$$
\begin{aligned}
& \frac{d^{2} X}{d \eta^{2}}+\left[A_{X}-2 Q_{X} \cos 2 \eta\right] X=0 \\
& \frac{d^{2} X}{d \eta^{2}}+\left[A_{Y}-2 Q_{Y} \cos 2 \eta\right] Y=0 .
\end{aligned}
$$

Here

$$
\begin{aligned}
& A_{X}=\frac{1}{\nu^{2}}\left(a+\frac{q^{2}}{2}\right), \quad A_{Y}=\frac{1}{\nu^{2}}\left(-a+\frac{q^{2}}{2}\right), \\
& Q_{X}=-Q_{Y}=-\frac{q^{\prime}}{\nu^{2}}
\end{aligned}
$$

The stability of the ion motion with quadrupole excitation is described by the Mathieu equations (eq 32). The usual stability diagram is not useful in practice in this case, because $A_{X}$ and $A_{Y}$ depend on ion mass in a complicated manner $\left(\mathrm{a} \sim 1 / \mathrm{m}\right.$ and $\left.q \sim 1 / \mathrm{m}^{2}\right)$. However this can be simplified for an RF only quadrupole. With $a=0$ one has $A_{X}=A_{Y}=A$ and the parameter $A$ is inversely proportional to the square of the ion mass. Hence the value of $\sqrt{A} / Q$ is independent of mass. It follows from this discussion, that the most appropriate parameters for the stability diagram are $\sqrt{A} / Q$. The Ince diagram for the Mathieu equation in the $\sqrt{A} / Q$ plane is shown in Figure 12a. This diagram can be used in the same manner as the diagram for normal quadrupole operation equation because the scan line $\sqrt{A}=\lambda \cdot Q$ has the same slope $\lambda$ for ions with different mass:

$$
\lambda=\frac{\nu q}{\sqrt{2} q^{\prime}}=\frac{\omega V}{\sqrt{2} \Omega V_{e x}}
$$

One must keep in mind that this diagram is limited to an RF-only quadrupole. If even a small DC voltage is added to the quadrupole field, then $A_{X}$ and $A_{Y}$ are different. In this case the stability diagrams for $\mathrm{x}$ and $y$ motion have different shapes. The same consideration applies to the 3-D quadrupole field. With RF-only operation the axial frequency is approximately twice the radial frequency. It follows that the RF diagram of Figure 12a is not applicable to 3-D quadrupole ion traps.

The result is approximate because of the averaging procedure. The result may be checked by means of direct calculation with the matrix method. The proce- 
(a)

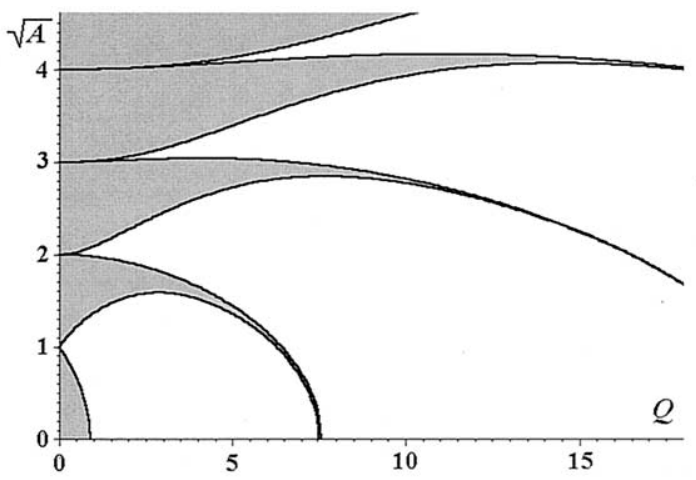

(b) $q$

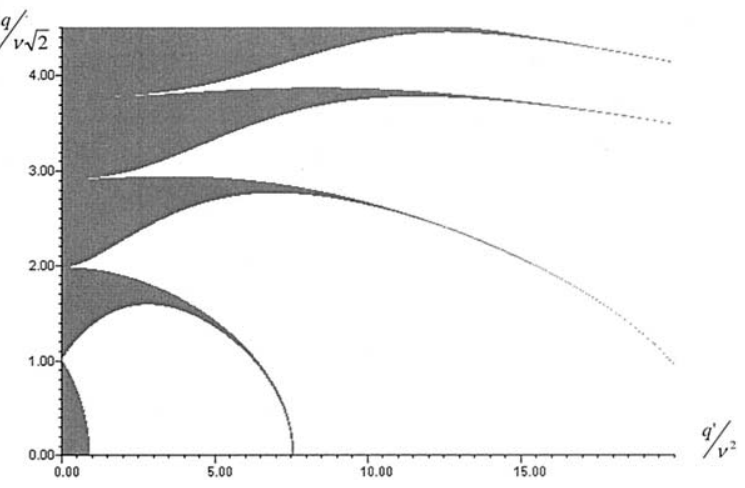

Figure 12. (a) Direct redrawing of the Ince diagram in the $(a, q)$ plane. (b) The stability diagram of the eq 26 for the case of $\nu=$ $1 / 10$ calculated by the matrix method.

dure is the same as described previously for the calculation of the diagram with quadrupole excitation. The difference is that the diagram is drawn in the plane of the $\left(q / \nu \sqrt{2}, q^{\prime} / \nu \sqrt{2}\right)$ parameters. The result of such a calculation for the case $\nu=1 / 10$ is shown in Figure 12b. Although the details of Figure 12a and b are a bit different, the qualitative structure of the diagrams is the same. Previous theoretical approaches to quadrupole excitation in quadrupole fields [6c, 21, 27] were based on perturbation theory for small values of $a$ and $q$. The perturbation result is valid if the parameter $Q$ is small. It requires the following conditions:

$$
Q \ll 1 \text {, or } \quad q^{\prime} \ll \nu^{2} \text { or } \quad \frac{4 Z e}{M \omega^{2} r_{0}^{2}} V_{e x} \ll 1
$$

For a small excitation frequency $\nu$ this condition significantly restricts the area of application. The matrix calculation which gives Figure $12 \mathrm{~b}$ is not limited to small parameters. The quadrupole excitation may be of any value, even much higher than the trapping voltage. For the case of $V=0$ (no main trapping voltage) the scan line is positioned along the $Q$ axis. In the absence of trapping voltage the auxiliary quadrupole field becomes the usual RF trapping field of frequency $\omega$ and voltage $V_{\text {ex }}$. According to definition (eq 33) the parameter $Q$ has the exact meaning as the $q$ value for this quadrupole field. It follows from Figure 12 that in the case $A=0$ the first stable area is at $Q<0.908$, the second stable area is near $Q=7.6$. This result is consistent with previous calculations of higher regions of stability, shown in Figure 7a and b and demonstrates the non-perturbative character of the calculations leading to Figure 12.

\section{Stability Diagram with Amplitude Modulation of the Trapping Voltage}

A periodic change of the main trapping RF voltage produces resonance lines in the stability diagram. Methods other than quadrupole excitation such as modulation of the RF amplitude [27] can produce similar results. With amplitude modulation at frequency $\omega$, the equation of ion motion is

$$
u^{\prime \prime}+[a-2 q \cos 2 \xi] \cdot[1+m \cos (2 \nu \xi+\alpha)] \cdot u=0 .
$$

Here $m$ is the modulation parameter, and $\nu=\omega / \Omega$ is the relative modulation frequency. The resulting trapping voltage function in eq 36 can be expressed as

$$
\begin{aligned}
& a-2 q \cos 2 \xi+a m \cdot \cos (2 \nu \xi+\alpha)-2 m q \cdot \cos 2 \xi \\
& \cdot \cos (2 \nu \xi+\alpha)=a-2 q \cos 2 \xi+m\{a \cdot \cos (2 \nu \xi+\alpha) \\
& -q \cdot \cos [2(1+\nu) \xi+\alpha]-q \cdot \cos [2(1-\nu) \xi-\alpha]\}
\end{aligned}
$$

From eq 37 it follows that modulation of the RF amplitude may be considered as simultaneous quadrupole excitation with two frequencies $1-\nu$ and $1+\nu$, and also an additional signal at the frequency $\nu$.

For the case of modulation with a rational frequency $\nu=\omega / \Omega=N / P$ the stability of ion motion may be investigated by the same methods. Several calculated stability diagrams with a fixed modulation parameter, $m=0.1$, are shown in Figure 13. The number of stable bands is the same as for the case of simple quadrupole excitation with one rational frequency.

\section{Discussion}

This paper describes the theory for calculating the stability of ion motion in pure quadrupole fields with periodic time varying potentials. The method of stability diagram calculation is based completely on matrix methods. This method allows calculating the diagram in the case of rectangular trapping waveforms (Figures 1-3). It is used to calculate the well known Ince diagram for the Mathieu equation (Figure 4) and the known general stability diagrams for an ion trap (Figure 5) and a mass filter (Figure 6). It can also be used to calculate higher regions of stability of a mass filter (Figures 7 and 8). Region $V$ is shown here for the first time.

The calculation of stability diagrams with auxiliary 
(a)

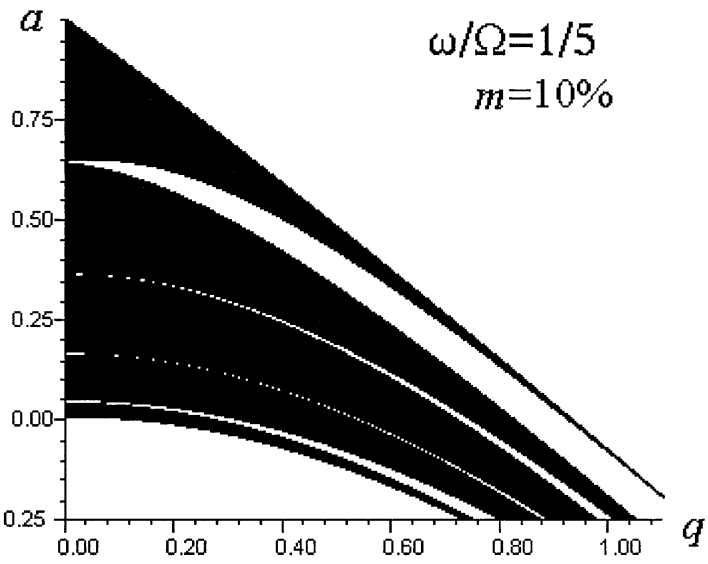

(b)

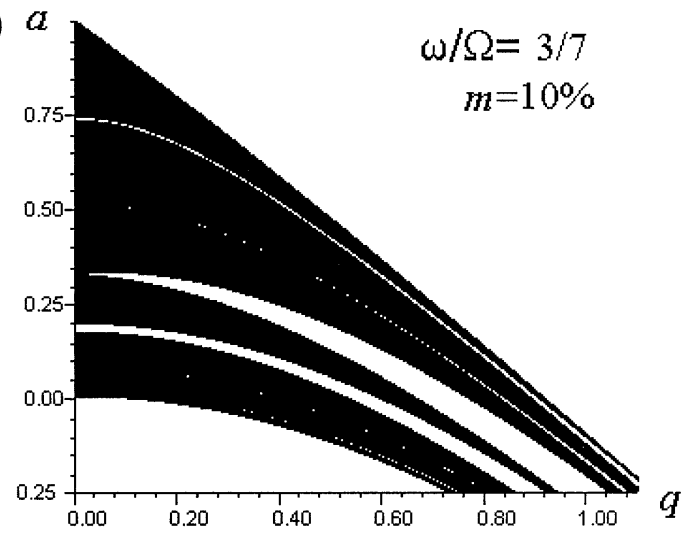

(c)

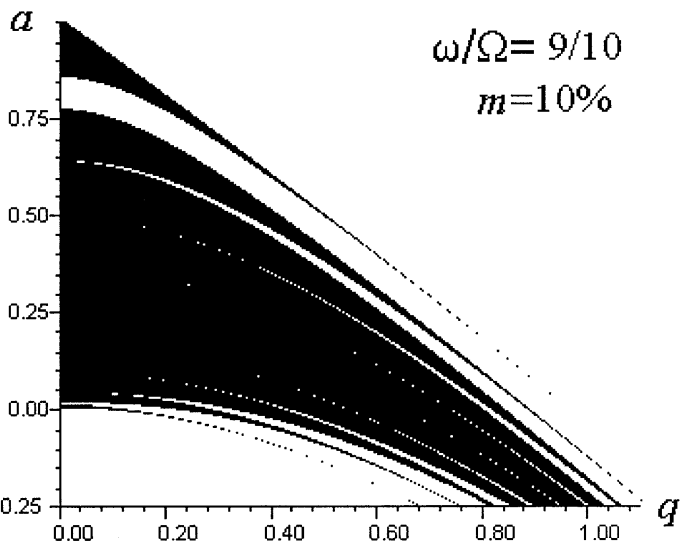

Figure 13. Stability diagram sections for the case of amplitude modulation with modulation parameter $m=0.10$ and frequency (a) $\omega=\Omega / 5$, (b) $\omega=3 \Omega / 7$, (c) $\omega=9 \Omega / 10$. Black regions have stable motion.

quadrupole excitation and modulation is described in detail for the first time. The results of these calculations show that with a periodic change of the RF voltage, the stable regions split into stable bands. The number of stable bands is $P$, the general multiple of the RF frequency and the auxiliary periodic excitation frequency. With low excitation amplitudes the resonance lines occur along iso- $\beta$ lines of the unperturbed diagram. The widths of the resonance lines increase with the excitation amplitude. The comparative widths of different resonance lines depend strongly on the excitation frequency, the excitation waveform and the excitation method. Each case needs independent investigation.

Unstable resonance lines and splitting of the stability diagram are general phenomena with auxiliary periodic changes in the main trapping RF. This follows from abstract mathematical models [28]. From the point of view of vibration theory, it is the usual manifestation of parametric resonance in a system with complicated vibration spectra, as is the case for ion motion in a periodic quadrupole field. The most general condition of parametric resonance, for ions in a periodic sinusoidal trapping field [21], is

$$
\begin{aligned}
& K \omega=\Omega|n+\beta|, \quad n=0, \pm 1, \pm 2, \ldots \\
& K=1,2, \ldots
\end{aligned}
$$

Here $n$ is an integer, $K$ is the order of a resonance, and $\Omega$ is the main trapping frequency. It is evident from eq 38 that for the case of a rational excitation frequency $\omega=N \Omega / P$ the values of $\beta=K / P, K=1,2, \ldots, P-1$ will satisfy the resonance condition. Equation 38 has a simple physical meaning: Resonance appears in a process when the energy of $K$ excitation quanta goes into the energy of two ion oscillation quanta. Equation 38 does not contain information about the resonance strength or about the width of a resonance line. The actual probability of the process depends on the excitation amplitude, on amplitudes of the corresponding Fourier spectrum harmonics, and also on the phase relations. In spite of the condition in eq 38, a resonance may not occur, if other conditions prevent it. (For example, if the excitation amplitude is below threshold for a damped oscillator).

This article considers only the case of rational excitation frequencies $\nu=N / Q$. The matrix method is not applicable to the case of an irrational frequency ratio, $\nu_{0}$, because the voltage waveform is not periodic. However, this work gives a qualitative approach to the description of the general case. Consider the series of rational frequencies $\nu_{1}=N_{1} / P_{1}, \nu_{2}=N_{2} / P_{2}, \nu_{3}=$ $N_{3} / P_{3}, \ldots$ Each of these numbers is closer and closer to the irrational number $\nu_{0}$, and denominators go in increasing order: $P_{1}<P_{2}<P_{3}<\ldots$. The present investigation proves that the diagram of stability will split into a number of stable bands. The number of unstable resonance lines in each case is equal to $P_{1}, P_{2}$, $P_{3} \ldots$. Most of these resonance lines are very weak. They will not appear in a practical experiment because of damping and nonlinear shifts of ion oscillation frequencies. Only the strongest resonance lines will survive. Hence, in practical experiments we see a limited number of resonances instead of the infinite series which is predicted by eq 38 .

In general the width of the splitting of a diagram is greater near the boundaries of stable regions compared 
to the width of resonance lines near the center of a stable region. An exception to this rule can be seen in Figure 13 for the case of amplitude modulation with frequency $\omega=\Omega / 7$. In this case the phase relations between the three excitation harmonics in eq 37 lead to some kind of forbidding of the resonances $\beta=1 / 7,2 / 7$, $5 / 7,6 / 7$. These resonance lines are present, but the widths are much smaller compared to the width of the main resonance at $\beta=3 / 7$ and $4 / 7$. The $\beta=3 / 7$ and $4 / 7$ resonances are the $\mathrm{K}=1$ resonances for $n=0$ and $n=$ 1 in eq 38. The $\beta=1 / 7,2 / 7,5 / 7$, and $6 / 7$ resonances require higher combinations of $n$ and $K$. Experiments show these have higher threshold voltages [29] and this may explain why they are weak in Figure 13. For the other cases the diagram splitting is stronger near the boundaries, especially when the excitation frequency is small or close to the RF frequency. This phenomena helps to explain the requirement for the high quality of supply voltages in quadrupole mass spectrometry devices.

In practice ion motion occurs in the presence of a buffer gas and the field may contain other multipoles and is different from that of a pure quadrupole. If the motion of assemblies of many ions is considered, then the influence of space charge must be considered. Collisions lead to damping of ion oscillations. With damping, quadrupole excitation has a threshold [12c, $20,30]$. Resonance does not appear if the excitation voltage is smaller than the threshold (in this case the amplitude of ion motion decreases exponentially). The positions of resonance lines depend on the excitation amplitude. The actual resonant frequency may differ slightly from that predicted by eq 38, when the excitation voltage is increased above threshold. This has been observed experimentally [25]. Higher order field imperfections lead to nonlinearity of ion oscillations. As a result, the ion oscillation frequency depends on amplitude. Space charge can also cause significant shifts in the ion oscillation frequency. The combination of nonlinearity and the space charge can lead to complicated shifts of resonance frequencies. Coulomb interactions may also cause collective phenomena that were described more than 30 years ago by Rettinghaus [31]. The equation of motion of the center of mass of an ion cloud does not contain internal ion-ion Coulomb forces and the mass to charge ratio of the ion cloud is the same as that of a single ion (provided ions of only one $\mathrm{m} / \mathrm{z}$ comprise the cloud). This equation of motion for the center of mass is the same as the equation of motion for a single ion in the absence of space charge. Collective parametric resonance has been observed under high vacuum conditions, when the energy of chaotic ion motion is small compared to the trapping well depth [32]. The above discussion and recent experiments indicate that the phenomena of parametric resonance of trapped ions is complex. The practical use of these phenomena will require further theoretical and experimental research.

\section{Acknowledgments}

MS gratefully acknowledges SCIEX, Concord, Ontario, Canada for financial support during a visit to the University of British Columbia. DJD acknowledges support by the Natural Sciences \& Engineering Research Council and SCIEX through an Industrial Chair.

\section{Appendix 1}

The theory of the stability of ion motion, determined by matrix methods, is described in this appendix. The application to periodic focusing systems and a more detailed mathematical description can be found in references [12b] and [12c] respectively.

For eq 4 with a periodic function, $f(\xi)$, the state vector representation is useful. An ion trajectory can be described by a sequence of state vectors $\left(x_{n}, v_{n}\right), n=$ $0,1, \ldots$ where $x_{n}$ and $v_{n}$ are the position and velocity after $n$ cycles. The sequence is defined by the matrix recurrence relation, eq 10. An ion trajectory is stable if, and only if, the sequence of state vectors is stable, that is, if $x$ remains finite. The recurrence eq 10 can be solved explicitly. The matrix $\mathrm{M}$ has $\operatorname{det}(\mathrm{M})=1$. Hence, as a real matrix, $M$ has exactly two eigenvalues, $\lambda_{1}$ and $\lambda_{2}$, and two eigenvectors $m_{1}$ and $m_{2}$ which obey the equations:

$$
\mathrm{M} m_{1}=\lambda_{1} m_{1}, \quad \mathrm{M} m_{2}=\lambda_{2} m_{2}
$$

The initial state vector $\left(x_{0}, v_{0}\right)$ can be expressed in terms of the eigenvectors $m_{1}$ and $m_{2}$ as

$$
\left(\begin{array}{c}
x_{0} \\
v_{0}
\end{array}\right)=C_{1} m_{1}+C_{2} m_{2}
$$

Here $C_{1}$ and $C_{2}$ are constants, which can be found from eq I. 2 once the eigenvectors $m_{1}$ and $m_{2}$ are calculated.

From the recurrence eq 10, with the use of eq I.2 and eq I.1 it follows that

$$
\begin{gathered}
\left(\begin{array}{l}
x_{1} \\
v_{1}
\end{array}\right)=\mathrm{M}\left(\begin{array}{c}
x_{0} \\
v_{0}
\end{array}\right)=\mathrm{M}\left(\mathrm{C}_{1} m_{1}+C_{2} m_{2}\right) \\
=C_{1} \mathrm{M} m_{1}+C_{2} \mathrm{M} m_{2}=C_{1} \lambda_{1} m_{1}+C_{2} \lambda_{2} m_{2} \\
\left(\begin{array}{l}
x_{2} \\
v_{2}
\end{array}\right)=\mathrm{M}\left(\begin{array}{c}
x_{1} \\
v_{1}
\end{array}\right)=\mathrm{M}\left(C_{1} \lambda_{1} m_{1}+C_{2} \lambda_{2} m_{2}\right) \\
=C_{1} \lambda_{1}^{2} m_{1}+C_{2} \lambda_{2}^{2} m_{2} \\
\left(\begin{array}{l}
x_{n} \\
v_{n}
\end{array}\right)=C_{1} \lambda_{1}^{n} m_{1}+C_{2} \lambda_{2}^{n} m_{2}
\end{gathered}
$$

It follows from eq I.3 that the sequence of state vectors $\left(x_{n}, v_{n}\right)$ will be stable if and only if the absolute values of both $\lambda_{1}$ and $\lambda_{2}$ are less than or equal to unity i.e.,

$$
\left|\lambda_{1,2}\right| \leq 1
$$


Eq I.4 is the stability condition for ion motion in terms of the eigenvalues of the transmission matrix. From this, we can derive the stability condition in terms of the elements of the matrix.

In order to find the eigenvectors write eq I.1 as follows

$$
\begin{aligned}
& {\left[\begin{array}{ll}
m_{11} & m_{12} \\
m_{21} & m_{22}
\end{array}\right] \cdot\left(\begin{array}{c}
x \\
y
\end{array}\right)=\lambda\left(\begin{array}{l}
x \\
y
\end{array}\right)} \\
& \text { or }\left\{\begin{array}{l}
\left(m_{11}-\lambda\right) x+m_{12} y=0 \\
m_{21} x+\left(m_{22}-\lambda\right) y=0
\end{array}\right.
\end{aligned}
$$

Here $x$ and $y$ are the unknown elements of an eigenvector, which are to be found from eq I.5. The latter is a system of linear equations with zero on the right side. It will have nonzero solutions if and only if the determinant of the system is equal to zero:

$$
\begin{aligned}
& \operatorname{det}\left[\begin{array}{cc}
m_{11}-\lambda & m_{12} \\
m_{21} & m_{22}-\lambda
\end{array}\right]=0, \text { or } \\
& \lambda^{2}-\left(m_{11}+m_{22}\right) \lambda+m_{11} m_{22}-m_{12} m_{21}=0 .
\end{aligned}
$$

An additional simplification of eq I.6 follows from the condition $\operatorname{det}(\mathrm{M})=m_{11} m_{22}-m_{12} m_{21}=1$. eq I. 6 can then be solved explicitly as follows:

$$
\lambda_{1}=s+i \sqrt{1-s^{2}}, \quad \lambda_{2}=s-i \sqrt{1-s^{2}}
$$

where

$$
s=\frac{m_{11}+m_{22}}{2}=0.5 \operatorname{Tr}(\mathrm{M})
$$

and $i=\sqrt{-1}$. Note that $\lambda_{1} \lambda_{2}=1$. If $|s|<1$ then both eigenvalues are complex numbers with unit absolute value: $\left|\lambda_{1}\right|=\left|\lambda_{2}\right|=1$. With complex eigenvalues we can still use eq 10, eq I.2, and eq I.3. In this case we can consider the actual solution for an ion trajectory to be the real part of a complex solution. This is possible because eq 10 is linear with respect to the state vector (the matrix $\mathrm{M}$ does not depend on the state vector).

In terms of the matrix elements the stability condition can be written as:

$$
|\operatorname{Tr}(\mathrm{M})|<2 \quad \text { or } \quad\left|m_{11}+m_{22}\right|<2 .
$$

Note from eq I.7 that the eigenvalues are complex conjugates, $\lambda_{1}{ }^{*}=\lambda_{2}$. Writing $\lambda_{1}=A e^{i \theta}$ we have $A=1$ and $\cos (\theta)=m_{1}+m_{2} / 2$. It is useful to introduce the parameter $\beta=\theta / \pi$ and so $\beta$ is given by

$$
\cos (\pi \beta)=s=\frac{m_{11}+m_{22}}{2} .
$$

Once the eigenvalues are found, we can calculate the eigenvectors from eq I.5. Then we can calculate coefficients $C_{1}$ and $C_{2}$ from eq I.2. Once this is done, we can use eq I. 3 to calculate the state vector after any number of periods $n$. The equation for the $n$-th power of matrix $M$ (see eq 11 in the paper) is a result of this procedure.

It is useful to discuss unstable motion of ions. For the case $s=1$ the ion motion is exactly on the stability boundary. As noted,

$$
\lambda_{1} \lambda_{2}=1, \quad \text { or } \quad \lambda_{1}=\frac{1}{\lambda_{2}} \text {. }
$$

For the case of $s>1$ the eigenvalues are real numbers. It follows from eq I.10 that if one eigenvalue is greater than one, the other is less than one. Assume that $\left|\lambda_{1}\right|>$ 1. Then, according to eq I.3, the coordinates of the ion increase exponentially: $x_{n} \propto C \lambda_{1}^{n}$. An exception to this is the special set of initial conditions $\left(x_{n}{ }^{*}, v_{n}{ }^{*}\right)$ for which the coefficient $C_{1}$ in eq I.3 happens to be exactly zero. Such initial conditions are unusual and consist of only a single line in the two dimensional space of all possible initial conditions. Even a very small departure from these special initial conditions results in an exponential increase of the ion trajectory. The rate of increase is determined by the increment $\mu=\ln \left(\lambda_{1}\right)$. It is usually defined by the following equation:

$$
\cosh (\mu)=\frac{\left|m_{11}+m_{22}\right|}{2}
$$

which is similar to eq I.4. The parameter $\mu$ has the physical meaning of the exponential increment in one RF cycle. The amplitude of the ion oscillation increases by a factor of $\exp (\mu)$ in each RF period. The kinetic energy of the ion increases by a factor of $\exp (2 \mu)$. This is a consequence of parametric resonance because in this process the energy gain is proportional to the energy of the ion vibration.

\section{References}

1. Ince, E. Researches into the Characteristic Numbers of the Mathieu Equation. Proceedings of the Royal Society of Edinburgh; Neiland Co. Ltd.: Edinburgh, 1925-1926; XLVI, 20-29.

2. McLachlan, N. W. Theory and Applications of Mathieu Functions; Clarendon: Oxford, 1947.

3. Quadrupole Mass Spectrometry and its Applications; Dawson, P. H., Ed.; Elsevier: Amsterdam, 1976; reissued by AIP Press: Woodbury, NY, 1995, pp 65-77.

4. March, R. E., Hughes, R. J., Todd, J. F. J. Quadrupole Storage Mass Spectrometry, Wiley Interscience, 1989, pp 31-73.

5. (a) Dawson, P. H.; Yu, B. The Second Stability Region of the Quadrupole Mass Filter. II. Experimental Results. Int. J. Mass Spectrom. Ion Proc. 1984, 56, 41-50. (b) Konenkov, N. V.; Kratenko, V. I. Characteristics of a Quadrupole Mass Filter in the Separation Mode of a Few Stability Regions. Int. J. Mass Spectrom. Ion Proc. 1991, 108, 115-136. (c) Du, Z.; Douglas, D. J.; Konenkov, N. V. Elemental Analysis with Quadrupole Mass Filters Operated in Higher Stability Regions. J. Anal. Atom. Spectrom. 1999, 14, 1111-1119. 
6. (a) Paul, W.; Reinhard, H. P.; Von Zahn, U. Das Elektrische Massenfilter als Massenspectrometer und Isotopentrenner. $Z$. Phys. 1958, 152, 143-182. (b) Vedel, F.; Vedel, M.; March, R. E. New Schemes for Resonant Ejection in RF Quadrupolar Ion Traps. Int. J. Mass Spectrom. Ion Proc. 1990, 99, 125-138. (c) Alfred, R. L.; Londry, F. A.; March, R. E. Resonance Excitation of Ions Stored in a Quadrupole Ion Trap. Part IV. Theory of Quadrupolar Excitation. Int. J. Mass Spectrom. Ion Proc. 1993, 124, 171-185. (d) Makarov, A. Resonance Ejection from the Paul Trap: A Theoretical Treatment Incorporating a Weak Octopole Field. Anal. Chem. 1996, 68, 4257-4263.

7. (a) Richards, J. A.; Huey, R. M.; Hiller, J. A New Operating Mode for the Quadrupole Mass Filter. Int. J. Mass Spectrom. Ion Proc. 1973, 12, 317-339. (b) Sheretov, E. P.; Terentyev, V. A. The Fundamentals of Theory of Quadrupole Mass Spectrometry with Pulsed Voltage Waveform. Russ. J. Tech. Phys. 1972, 42, 953-962.

8. Floquet, G. Sur les Equations Differentielles Lineares a Coefficients Periodiques. Ann. Ecole Norm. Sup. Paris 1883, 12, 47-89.

9. (a) Hale, J. K. Ordinary Differential Equations; Wiley Interscience: New York, 1969, pp 117-131. (b) Hartman, P. Ordinary Differential Equations; 2nd ed., Burkhauser, Boston, 1982, pp 60-62.

10. Pipes, L. A. Matrix Solution of Equations of the Mathieu-Hill Type. J. Appl. Phys. 1953, 24, 902-910.

11. Waldren, R. M.; Todd, J. F. J. The Use of Matrix Methods and Phase-Space Dynamics for the Modelling of Quadrupole-Type Device Performance. In Dynamic Mass Spectrometry; Price, D.; Todd, J. F. J., Eds.; Heyden: London, 1978; Chap V; 14-40.

12. (a) Dawson, P. H. Numerical Calculation. In Quadrupole Mass Spectrometry and its Applications. Elsevier: Amsterdam, 1976; 84. (b) Humphries, S., Jr.; Principles of Charged Particle Acceleration; John Wiley and Sons: New York, 1986. (c) Arnol'd, V. I. Ordinary Differential Equations, 3rd ed.; Springer-Verlag: Berlin, 1991; pp 256-264.

13. Symon, K. R. Mechanics. Addison-Wesley Publishing Co. Inc.: Reading, 1964; p 399.

14. Ding, L.; Kumashiro, S. Ion Motion in the Rectangular Wave Quadrupole Field and Digital Operation Mode of a Quadrupole Mass Spectrometer. Chinese Vac. Sci. Tech. 2001, 3, 176181.

15. Sudakov, M. Stability Diagrams of Ions in Radio Frequency Mass Spectrometry. Russ. J. Tech. Phys. 1994, 65, 170-178.

16. (a) Kaiser, R. E., Jr.; Cooks, R. G.; Stafford, G. C.; Syka, J. E. P.; Hemberger, P. H. Operation of a Quadrupole Ion Trap Mass Spectrometer to Achieve High Mass/Charge Ratios. Int. J. Mass Spectrom. Ion Proc. 1991, 106, 79-115. (b) Schwartz, J. C.; Syka, J. E. P.; Jardine, I. High Resolution in a Quadrupole Ion Trap Mass Spectrometer. J. Am. Soc. Mass Spectrom. 1991, 2, 198-204. (c) Londry, F. A.; Wells, G. J.; March, R. E. Enhanced Mass Resolution in a Quadrupole Ion Trap. Rapid Commun. Mass Spectrom. 1993, 7, 43-45.

17. Sevugarajan, S.; Menon, A. G. Frequency Perturbation in Nonlinear Paul Traps: A Simulation Study of the Effect of Geometric Aberration, Space Charge, Dipolar Excitation, and Damping on Ion Axial Secular Frequency. Int. J. Mass Spectrom. 2000, 197, 263-278.
18. Alheit, R.; Kleineidam, S.; Vedel, F.; Vedel, M.; Werth, G. Higher Order Non-Linear Resonances in a Paul Trap. Int. J. Mass Spectrom. Ion Proc. 1996, 154, 155-169.

19. Chen, W.; Collings, B. A.; Douglas, D. J. High-Resolution Mass Spectrometry with a Quadrupole Operated in the Fourth Stability Region. Anal. Chem. 2000, 72, 540-545.

20. (a) Landau, L. D.; Lifshitz, E. M. Mechanics, 3rd ed. Pergamon Press: Oxford, 1960, pp 80-83. (b) Bogolubov, N. N.; Mitropol'skii, Y. A. Problems of the Asymptotic Theory of Nonstationary Vibrations; Israel Program for Scientific Translations: Jerusalem, 1965.

21. Sudakov, M.; Konenkov, N.; Douglas, D. J.; Glebova, T. Excitation Frequencies of Ions Confined in a Quadrupole Field With Quadrupole Excitation. J. Am. Soc. Mass Spectrom. 2000, 11, 11-18.

22. Kozo, M. Quadrupole Mass Spectrometer, US patent 5,227,629, July 13, 1993.

23. (a) Konenkov, N. V.; Cousins, L. M.; Baranov, V. I.; Sudakov, M. Y. Quadrupole Mass Filter Operation with Auxiliary Quadrupolar Excitation: Theory and Experiment. Int. J. Mass Spectrom 2001, 208, 17-27. (b) Baranov, V. I.; Konenkov, N. V.; Tanner, S. D. QMF Operation with Quadrupole Excitation. In Plasma Source Mass Spectrometry, the New Millenium; Holland, G.; Tanner, S. D., Eds.; Royal Society of Chemistry: Cambridge, 2001, pp 63-72.

24. Du, Z.; Olney, T.; Douglas, D. J. Inductively Coupled Plasma Mass Spectrometry with a Quadrupole Mass Filter Operated in the Third Stability Region. J. Am. Soc. Mass Spectrom. 1997, 8, 1230-1236.

25. (a) Collings, B. A.; Douglas, D. J. Observation of Higher Order Quadrupole Excitation Frequencies in a Linear Ion Trap. J. Am. Soc. Mass Spectrom. 2000, 11, 1016-1022. (b) Collings, B. A.; Sudakov, M.; Londry, F. Resonance Shifts in the Excitation of the $n=0, K=1$ to 6 Quadrupolar Resonances for Ions Confined in a Linear Ion Trap. J. Am. Soc. Mass Spectrom., in press.

26. Sudakov, M. Stability Diagram of Secular Motion of Ions Trapped in RF Quadrupole Field with Auxiliary Harmonic Excitation (in Russian). Pisma JTP 2000, 26, 46-51.

27. Sheretov, E. P.; Gurov, V. S.; Kolotilin, B. I. Modulation Parametric Resonances and Their Influence on Stability Diagram Structure. Int. J. Mass Spectrom. 1999, 184, 207-216.

28. Arnol'd, V. I. Comments on Perturbation Theory for MathieuLike Problems. Russ. Uspehi Math. Sci. 1983, 38, 189-203.

29. Cha, B.; Sudakov, M.; Douglas, D. J. unpublished.

30. (a) Razvi, M. A.; Chu, X. Z.; Alheit, R.; Werth, G.; Blumel, R. Fractional Frequency Collective Parametric Resonance of an Ion Cloud in a Paul Trap. Phys. Rev. A 1998, 58, R34-R37. (b) Chu, X. Z.; Holzki, M.; Alheit, R.; Werth, G. Observation of High-Order Motional Resonances of an Ion Cloud in a Paul Trap. Int. J. Mass Spectrom. Ion Proc. 1998, 173, 107-112.

31. Rettinghaus, G. Ph.D. Thesis, Bonn University, 1965, unpublished.

32. Alheit, R.; Chu, X. Z.; Hofer, M.; Holzki, M.; Werth, G.; Blumel, R. Nonlinear Collective Oscillations of an Ion Cloud in a Paul Trap. Phys. Rev. A 1997, 56, 4023-4031. 